\title{
Determining Excited-State Structures and Photophysical Properties in Phenylphosphine Rhenium(I) Diimine Biscarbonyl Complexes Using Time- Resolved Infrared and X-ray Absorption Spectroscopies
}

Yuushi Shimoda ${ }^{1}$, Kiyoshi Miyata ${ }^{1}$, Masataka Funaki ${ }^{2}$, Tatsuki Morimoto ${ }^{3}$, Shunsuke Nozawa ${ }^{4, *}$, Shin-ichi Adachi ${ }^{4}$, Osamu Ishitani ${ }^{2}$, and Ken Onda ${ }^{1, *}$

${ }^{1}$ Department of Chemistry, Faculty of Science, Kyushu University, Motooka, Nishi-ku, Fukuoka 819-0395, Japan

${ }^{2}$ Department of Chemistry, School of Science, Tokyo Institute of Technology, O-okayama, Meguro-ku, Tokyo 152-8551, Japan

${ }^{3}$ Department of Applied Chemistry, School of Engineering, Tokyo University of Technology, Katakuramachi, Hachioji City, Tokyo 192-0982, Japan.

${ }^{4}$ Photon Factory, Institute of Materials Structure Sciences, High Energy Accelerator Research Organization (KEK), Oho, Tsukuba, Ibaraki 305-0801 Japan

*Corresponding authors: konda@chem.kyushu-univ.jp,noz@post.kek.jp 


\begin{abstract}
:
We have explored the structural factors on the photophysical properties in two rhenium(I) diimine complexes in acetonitrile solution, cis,trans- $\left[\operatorname{Re}(\mathrm{dmb})(\mathrm{CO})_{2}\left(\mathrm{PPh}_{2} \mathrm{Et}\right)_{2}\right]^{+}(\mathrm{Et}(2,2))$ and cis,trans$\left[\operatorname{Re}(\mathrm{dmb})(\mathrm{CO})_{2}\left(\mathrm{PPh}_{3}\right)_{2}\right]^{+}((3,3))\left(\mathrm{dmb}=4,4^{\prime}\right.$-dimethyl-2,2'-bipyridine, $\mathrm{Ph}=$ phenyl, Et = ethyl $)$ using the combination method of time-resolved infrared spectroscopy, time-resolved extended Xray absorption fine structure, and quantum chemical calculations. The difference between these complexes is the number of phenyl groups in the phosphine ligand, and this only indirectly affects the central $\operatorname{Re}(\mathrm{I})$. Despite this minor difference, the complexes exhibit large differences in emission wavelength and excited-state lifetime. Upon photoexcitation, the bond length of Re-P and angle of P-Re-P are significantly changed in both complexes, while the phenyl groups are largely rotated by $\sim 20^{\circ}$ only in $(3,3)$. In contrast, there is little change in charge distribution on the phenyl groups when Re to dmb charge transfer occurs upon photoexcitation. We concluded that the instability from steric effects of phenyl groups and diimine leads to the smaller Stokes shift of the lowest excited triplet state $\left(T_{1}\right)$ in $(3,3)$. The large structural change between the ground and excited states causes the longer lifetime of $\mathrm{T}_{1}$ in $(3,3)$.
\end{abstract}




\section{Introduction}

Rhenium(I) diimine biscarbonyl complexes that bear two phosphine ligands with various numbers of allyl groups or alkyl groups are widely known as efficient redox photosensitizers in photocatalytic systems for $\mathrm{CO}_{2}$ reduction [1-6]. These complexes are good building blocks for linear-shaped [7,8] or ring-shaped [9-12] $\operatorname{Re}(\mathrm{I})$ multinuclear complexes. Such complexes have been used in various applications as photofunctional materials such as light harvesting materials [8,13], efficient photosensitizers [9,12], and multielectron storage molecules [10]. A useful characteristic of these complexes for these applications is that their photophysical and photochemical properties are controllable by varying the number and type of functional groups of the two phosphine ligands $[4,6]$.

In general, the redox potentials, absorption, and emission wavelengths in these complexes are linearly dependent on the sum of the Tolman's $\chi$ values, which are an index of the electron accepting ability of phosphine ligands $[4,6]$. There are deviations from these trends when the complexes bear different numbers of aryl groups [6]. In particular, the Stokes shifts, which are the difference between absorption and emission energies, significantly decrease and the excited state lifetimes significantly increase when the complexes bare three aryl groups. These characteristics in the complexes bare three aryl groups are favorable for redox photosensitizers. In a previous report [6], these deviations were attributed to $\pi$ - $\pi$ interaction between the aryl groups of the two phosphine ligands and a diimine ligand. The $\pi$ - $\pi$ interaction is a generic concept that includes various interactions from attractive to repulsive forces between aromatic rings [14-17]. In the current study we examined the electronic and structural properties of two prototypical complexes in the excited-state using time-resolved infrared vibrational spectroscopy (TR-IR), time-resolved extended X-ray absorption fine structure (TR-EXAFS), and quantum chemical calculations.

TR-IR is a powerful tool for studying metal complexes in the excited state [18]. In particular, the IR spectra of double bonds such as $\mathrm{C}=\mathrm{C}$ and $\mathrm{C}=\mathrm{N}$ stretching vibrational modes in the fingerprint region are good probes for the electronic and structural character of aromatic groups in the excited state, for aromatic ligands in metal complexes [19-23], and organic compounds [24-27]. There are two major advantages to studying intramolecular interactions in molecules containing aromatic groups using TR-IR. One is that a geometry for the lowest excited triplet state $\left(T_{1}\right)$ can be determined in combination with quantum chemical calculations [20-23]. This is because $T_{1}$ is the lowest triplet state so optimized geometries can be calculated without considering configuration interactions or having to use time-dependent density functional theory (TD-DFT). This was confirmed using prototypical $\left[\mathrm{Ru}(\mathrm{bpy})_{3}\right]^{2+}$ and its ligand- and isotope-substituted complexes [20,23]. The other major advantage is that correlations between photophysical properties and structural dynamics can be derived from temporal evolutions of TR-IR spectra [19, 
21, 24-27]. The wavenumber and intensity of vibrational modes including double-bond vibrations are sensitive to the bond order and structure. Variations in bond order and structure can therefore be traced after photoexcitation in real-time. Using this method, we revealed that inter- and intramolecular interactions between aromatic groups play an important role for photophysical properties such as photoinduced phase transition materials [19, 24, 25], photoactive liquid crystals [26], and thermally-activated delayed fluorescence materials [27].

The lowest photoexcited state in $\operatorname{Re}(\mathrm{I})$ diimine complexes is the metal-to-ligand charge transfer (MLCT) state. Thus, the positions of atoms adjacent to the central $\operatorname{Re}(\mathrm{I})$ atom are key for understanding the structural dynamics upon photoexcitation. EXAFS is one of the best methods to determine the positions of atoms adjacent to metal atoms $[28,29]$. It has recently become possible to measure TR-EXAFS of a metal complex in solution in sub-nanosecond time resolution with high sensitivity comparable to that of TR-IR [30-36]. TR-EXAFS provides complementary information to TR-IR on the structure of metal complexes. This is because TR-IR spectra above $1000 \mathrm{~cm}^{-1}$ are sensitive to vibrations within ligands but not to vibrations between a central metal atom and ligands.

In this study, we focus on two $\operatorname{Re}(\mathrm{I})$ diimine biscarbonyl complexes that have very different photophysical properties despite their similar molecular structures. One is cis, trans$\left[\mathrm{Re}(\mathrm{dmb})(\mathrm{CO})_{2}\left(\mathrm{PPh}_{2} \mathrm{Et}_{2}\right]^{+}\left[\mathrm{PF}_{6}\right]^{-}\left(\mathrm{dmb}=4,4^{\prime}\right.\right.$-dimethyl-2,2'-bipyridine, $\mathrm{Ph}=$ phenyl, $\mathrm{Et}=$ ethyl $)$ $\left(\operatorname{Et}(2,2)\right.$, Figure 1a) and the other is cis, trans- $\left[\mathrm{Re}(\mathrm{dmb})(\mathrm{CO})_{2}\left(\mathrm{PPh}_{3}\right)_{2}\right]^{+}\left[\mathrm{PF}_{6}\right]^{-}((3,3)$, Figure 1b). According to the previous report [6], the absorption wavelengths of $\operatorname{Et}(2,2)$ and $(3,3)$ to ${ }^{1}$ MLCT (metal-to-ligand charge-transfer state) are almost the same at 403 and $402 \mathrm{~nm}$, respectively. However, their emission wavelengths from ${ }^{3}$ MLCT are very different at 622 and $600 \mathrm{~nm}$, respectively. This indicates that the Stokes shift of $(3,3), 8200 \mathrm{~cm}^{-1}$, is significantly smaller than that of $\operatorname{Et}(2,2), 8700 \mathrm{~cm}^{-1}$. The lifetime of ${ }^{3} \operatorname{MLCT}$ of $(3,3)$ is $1280 \mathrm{~ns}$, which is more than twice as long as that of $\operatorname{Et}(2,2)$ at 646 and 203 ns (dual emission). The higher energy (because of the small Stokes shift) and longer lifetime of ${ }^{3}$ MLCT of $(3,3)$ should make $(3,3)$ a better redox photosensitizer than $\operatorname{Et}(2,2)$.

To explore the difference in photophysical properties between $\operatorname{Et}(2,2)$ and $(3,3)$ in terms of excited-state character, we measured TR-IR spectra in the wavenumber range from 1000 to 2200 $\mathrm{cm}^{-1}$ and carried out spectral simulations using DFT calculations. To confirm the assignments of vibrational modes, we carried out measurements on deuterated $(3,3)$ in which all hydrogen atoms of phenyl groups were replaced by deuterium ((3D, 3D), Figure 1c). We measured TR-EXAFS under almost the same conditions as the TR-IR measurements, and analyzed the positions of atoms adjacent to $\operatorname{Re}(\mathrm{I})$. We discuss in detail the electronic and structural character based on geometries confirmed by TR-IR and TR-EXAFS. We found little difference in the electronic properties but a large difference in the excited-state structures between $\operatorname{Et}(2,2)$ and $(3,3)$, which 
should cause the difference in photophysical properties.

\section{Experimental}

2.1. Materials. All complexes were synthesized according to a previous report [6].

2.2. Instrumentation. Detailed procedures of steady-state IR and TR-IR measurements are reported elsewhere $[18,23]$. The steady-state IR spectra were measured by a Fourier-transform infrared (FT-IR) spectrometer (Shimadzu Prestage-21). KBr pellets for FT-IR measurements were prepared from $1.0 \mathrm{mg}$ of sample and $150 \mathrm{mg}$ of $\mathrm{KBr}$ powder. TR-IR spectra were acquired by a purpose-built system based on the pump-probe method, using a femtosecond Ti:sapphire chirped pulse amplifier (Spectra Physics Spitfire Ace, pulse duration $=120 \mathrm{fs}$, wavelength $=800 \mathrm{~nm}$, repetition rate $=1 \mathrm{kHz}$ ). The pump pulse (wavelength $=400 \mathrm{~nm}$, fluence at sample position $=7$ $\mathrm{mJ} / \mathrm{cm}^{2}$ ) was generated using the second harmonic generation of part of the amplifier output. The tunable probe pulse (wavenumber range $=1000-4000 \mathrm{~cm}^{-1}$, bandwidth $=150 \mathrm{~cm}^{-1}$ ) was obtained by an optical parametric amplifier equipped with a crystal for difference frequency generation (Lighconversion, TOPAS-Prime). The pump and probe pulses were irradiated on an infrared flow cell with $\mathrm{BaF}_{2}$ windows (optical path length $=0.1$ or $0.5 \mathrm{~mm}$ ). The polarizations of the pump and probe pulses on the flow cell were set to the magic angle using half wave plates for visible and mid-infrared wavelengths to avoid the effect of rotational relaxation. The spectrum of each probe pulse after passing through the flow cell was recorded by a mercury-cadmium-tellurium (MCT) infrared linear array system (Infrared Systems Development FpAS-64166-D). TR-IR spectra were obtained by averaging the difference between those with and without the pump pulse using an optical chopper synchronized with the pump pulse. The wavenumber resolution was $1-3 \mathrm{~cm}^{-1}$, which depends on wavenumber because of the linearity of polychromator. The accuracy of the wavenumber was calibrated by comparing to the spectrum of a polystyrene film to within $2 \mathrm{~cm}^{-}$ 1. Typical samples for TR-IR measurements were $3 \mathrm{mM}$ acetonitrile solutions, which were bubbled with nitrogen gas before and during each measurement. All measurements were carried out at room temperature.

TR-EXAFS experiments with the pump-probe method were conducted at the AR-NW14A beamline in the Photon Factory Advanced Ring (PF-AR) [37]. The pump pulse (wavelength = $343 \mathrm{~nm}$, fluence at sample position $=6 \mathrm{~mJ} / \mathrm{cm}^{2}$ ) was generated by the third harmonic generation of a femtosecond $\mathrm{Yb}$ fiber laser (Amplitude Tangerine). TR-EXAFS spectra were collected in fluorescence mode using a plastic scintillation detector. To measure the spectral change by the laser excitation precisely, the fluorescence X-ray signals before and after laser excitation were measured by gated integrators synchronized with the laser pulse $(397 \mathrm{kHz})$. The sample $(5 \mathrm{mM}$ acetonitrile solution) was circulated using a diaphragm pump to reduce radiation damage by the 
laser and X-ray, and was shaped to a stable $450-\mu \mathrm{m}$-thick jet using a stainless steel nozzle.

2.3. Quantum chemical calculations. All quantum chemical calculations were performed using the Gaussian16 software package [38]. The geometries of the ground state $\left(\mathrm{S}_{0}\right)$ and $\mathrm{T}_{1}$ were optimized by DFT calculation using the mPW1PW91 functional and the LanL2DZ basis set. Solvent effects of acetonitrile were considered using the conductor-like polarizable continuum model. The IR vibrational spectra of $S_{0}$ and $T_{1}$ were simulated on the basis of the optimized geometries, and difference spectra corresponding to experimental TR-IR spectra were obtained by subtracting the $S_{0}$ spectra from the $T_{1}$ spectra. To fit the calculated spectra to the experimental spectra, we considered the bandwidth $=10 \mathrm{~cm}^{-1}$ and a scaling factor $=0.97$ for vibrational modes at wavenumbers less than $1650 \mathrm{~cm}^{-1}$ [20] and a scaling factor $=1.02$ for CO stretching vibrational modes ranging from 1750 to $2050 \mathrm{~cm}^{-1}$ [39]. To discuss changes in charge distribution upon electronic transition by photoexcitation, we performed TD-DFT calculations and obtained natural transition orbitals (NTOs) [40].

\section{Results and Discussion}

3.1. Experimental and calculated spectra and vibrational mode assignments

Figures $2 \mathrm{a}$ and $2 \mathrm{c}$ show the FT-IR spectra and Figures $2 \mathrm{~b}$ and $2 \mathrm{~d}$ show the calculated spectra of $\operatorname{Et}(2,2)$ and $(3,3)$, respectively. The intensities less than $1650 \mathrm{~cm}^{-1}$ are shown enlarged for clarity. The FT-IR and calculated spectra are in good agreement with each other for both complexes, so we assigned the observed vibrational bands to the normal vibrational modes obtained by the calculations. The colors indicate the ligand at which the vibrations of each vibrational mode are mainly localized, judging from the view of the vibrational mode obtained by the calculations (Figure S1-S4). Peaks in blue, green, red, and gray are mainly localized at CO, $\mathrm{dmb}, \mathrm{Ph}$, and Et, respectively. Roughly speaking, the bands at $1850-1950 \mathrm{~cm}^{-1}$ are assigned to $\mathrm{CO}$ stretching vibrational modes while those at $<1650 \mathrm{~cm}^{-1}$ are assigned to $\mathrm{dmb}$ and $\mathrm{Ph}$.

Figures $3 \mathrm{a}$ and $3 \mathrm{c}$ show the TR-IR spectra at $100 \mathrm{ps}$ of $\operatorname{Et}(2,2)$ and $(3,3)$, respectively, and Figures $3 \mathrm{~b}$ and $3 \mathrm{~d}$ show their corresponding calculated spectra. These spectra show the difference in absorbance ( $\Delta$ abs) spectra between the ground and excited states. Upward and downward bands are assigned to vibrational transitions in the excited (transient absorption band) and ground states (bleach band), respectively. The TR-IR and calculated spectra are also in good agreement with each other for both complexes, so it is reasonable to discuss the character of $\mathrm{T}_{1}$ based on these calculations. In the same manner as above, we assigned the bands to normal vibrational modes and the colors indicate the same groups as those for the FT-IR results. There are two distinctive features in these spectra. One is that the wavenumbers of bands assigned to $\mathrm{CO}$ (indicated by blue) are increased by $40-70 \mathrm{~cm}^{-1}$ compared with the corresponding bleach bands. These blue 
shifts are well known and indicate that the charge at Re is reduced by the charge transfer from Re to dmb upon photoexcitation, and the $\mathrm{C}-\mathrm{O}$ bands are strengthened by a decrease in $\pi$ backdonation from $\operatorname{Re}$ [41-44]. The other distinctive feature is that almost all bands at wavenumber $<1650 \mathrm{~cm}^{-1}$ (indicated by green) except that at $1100 \mathrm{~cm}^{-1}$ (indicated by red) are assigned to vibrational modes of dmb, which is largely different from the ground state spectra. This feature strongly indicates that charge variations by the charge transfer occur mainly on dmb. This speculation is supported by the NTO analysis from DFT calculations, which is described later.

To confirm our vibrational mode assignments, we measured the TR-IR spectra and carried out spectral simulations of (3D,3D). The measured and calculated results are shown in Figure 4a and $4 \mathrm{~b}$, respectively. The normal mode vibrations assigned to $\mathrm{Ph}$ are strongly localized in $\mathrm{Ph}$ (Figure S4), so the deuteration of $\mathrm{Ph}$ only affects the vibrations in $\mathrm{Ph}$. The overall features of both the measured and calculated spectra are almost the same as those of $(3,3)$ (Figure S5), indicating that there are few bands assigned to vibrational modes localized in $\mathrm{Ph}$. However, carefully comparing the spectra of $(3,3)$ in Figure $3 \mathrm{c}$ and $(3 \mathrm{D}, 3 \mathrm{D})$ in Figure $4 \mathrm{a}$ reveals that the shapes of the bands assigned to $\mathrm{Ph}$ (indicated in red at approximately $1100 \mathrm{~cm}^{-1}$ in Figure $3 \mathrm{c}$ ) are different. To show this difference more clearly, the TR-IR spectra of $(3,3)$ and (3D,3D) are compared in the range from 1085 to $1130 \mathrm{~cm}^{-1}$ in Figure 4c. Although there is little difference in spectral shape at $1100-1120 \mathrm{~cm}^{-1}$, the transient absorption and bleach bands at $1085-1100 \mathrm{~cm}^{-1}$ are only observed in $(3,3)$. This indicates that these bands are assigned to vibrations localized in $\mathrm{Ph}$. This result strongly suggests that the bands at $1085-1100 \mathrm{~cm}^{-1}$ are a good probe for changes in the character of $\mathrm{Ph}$ upon photoexcitation.

\subsection{Temporal evolutions of TR-IR spectra}

Temporal evolutions of TR-IR are useful for understanding the electronic and structural character of ligands in photoexcited states. We measured temporal evolutions of the TR-IR spectra of $\operatorname{Et}(2,2)$ and $(3,3)$ in three wavenumber regions where typical bands assigned to each ligand are located: $1825-2050 \mathrm{~cm}^{-1}$ for CO (Figure 5), 1180-1260 $\mathrm{cm}^{-1}$ for dmb (Figure 6), and 1075-1120 $\mathrm{cm}^{-1}$ for Ph (Figure 7). In each figure, (a) and (b) show data of $\operatorname{Et}(2,2)$ and $(3,3)$, respectively. The effects of rotational relaxation on the spectra were canceled by the magic angle configuration.

In Figures 5a and 5b, the two bleach bands show little spectral change up to $100 \mathrm{ps}$, whereas the transient absorption bands show slight blueshift and bandwidth narrowing within $10 \mathrm{ps}$. These trends in spectral evolution are almost the same as those in Figures $6 \mathrm{a}$ and $6 \mathrm{~b}$. On the basis of the spectral simulations obtained by the quantum chemical calculations, the bands in Figures 5 and 6 are assigned to the vibrations purely localized on $\mathrm{CO}$ and $\mathrm{dmb}$, respectively. Except for the slight blueshift and narrowing, which result from vibrational relaxation in the excited states [41], the large wavenumber shifts of these transient absorption bands from those in the ground state occur 
less than 1 ps after photoexcitation. This indicates the charge transfer from Re to dmb occurs immediately after photoexcitation. No change in bleach bands up to $100 \mathrm{ps}$ indicates that there is no relaxation process to the ground state up to100 ps.

In contrast, the temporal evolutions of bands in Figure 7 are very different from those in Figures 5 and 6 . On the basis of the calculations, vibrational bands in this region largely contain vibrations of $\mathrm{Ph} . \mathrm{Ph}$ therefore has different dynamics from the other ligands in the early process after photoexcitation. The band at $1100 \mathrm{~cm}^{-1}$ in Figure $7 \mathrm{a}$, which consists of normal vibrational modes strongly localized on $\mathrm{Ph}$, increases gradually from 1 to $100 \mathrm{ps}$ though it starts at a negative value because of overlapping with a bleach band. The band at $1100 \mathrm{~cm}^{-1}$, which consists of vibrational modes of both $\mathrm{dmb}$ and $\mathrm{Ph}$, also increases gradually from 1 to $30 \mathrm{ps}$ after the sudden increase at 1 ps. In Figure 7b, the temporal behaviors of the bands are almost the same as those in Figure 7a, except that the overlapping between the transient absorption band and bleach band is much larger in the range from 1087 to $1097 \mathrm{~cm}^{-1}$.

To show the different dynamics of each ligand clearly, we plot the intensities of typical bands at $1971-1976 \mathrm{~cm}^{-1}$ for CO (blue squares), at $1212-1214$ and $1214-1217 \mathrm{~cm}^{-1}$ for $\mathrm{dmb}$ (green squares), and at 1098 and $1093 \mathrm{~cm}^{-1}$ for $\mathrm{Ph}$ (red squares), as a function of delay time in Figures $8 \mathrm{a}$ and $8 \mathrm{~b}$ for $\operatorname{Et}(2,2)$ and $(3,3)$, respectively. The intensities of the bands for $\mathrm{CO}$ and $\mathrm{dmb}$ were estimated by fitting to Gaussian functions to cancel the effects of the blueshift and narrowing. For the intensities of the bands for $\mathrm{Ph}$, the $\Delta \mathrm{Abs}$ at $1198 \mathrm{~cm}^{-1}$ for $\operatorname{Et}(2,2)$ and the $\Delta \mathrm{Abs}$ at $1193 \mathrm{~cm}^{-1}$ for $(3,3)$ were simply plotted because it is difficult to extract one band from the experimental spectra consisting of several transient absorption and bleach bands. For all three plots, the intensities change greatly less than $1 \mathrm{ps}$ after photoexcitation followed by a gradual increase over $10 \mathrm{ps}$ for $\mathrm{CO}$ and $\mathrm{dmb}$ and over $50 \mathrm{ps}$ for $\mathrm{Ph}$. The time constants of these gradual increases were estimated using a single exponential function. The estimated time constants for $\operatorname{Et}(2,2)$ in Figure $8 \mathrm{a}$ are $1.2 \pm 0.1 \mathrm{ps}$ for $\mathrm{CO}, 2.0 \pm 0.1 \mathrm{ps}$ for $\mathrm{dmb}$, and $30.8 \pm 1.4 \mathrm{ps}$ for $\mathrm{Ph}$, and those for $(3,3)$ in Figure $8 \mathrm{~b}$ are $1.2 \pm 0.1 \mathrm{ps}$ for $\mathrm{CO}, 3.7 \pm 0.3 \mathrm{ps}$ for $\mathrm{dmb}$, and $25.2 \pm 0.7 \mathrm{ps}$ for Ph. These results strongly indicate that phenyl groups in the phosphine ligands in both complexes do not undergo structural changes immediately after charge transfer from Re to dmb by photoexcitation. Rather, it takes more than 20 ps for their structural changes to occur after charge transfer.

\subsection{TR-EXAFS determination of atomic displacements adjacent to Re atom}

To determine directly the positions of atoms adjacent to the central Re(I) atom, we measured TR-EXAFS of $(3,3)$ at the Re-LIII absorption edge. Details of the analysis can be found in the supporting information. For $\mathrm{S}_{0}$ and $\mathrm{T}_{1}$, EXAFS spectra weighted by $k^{3}$ ( $k$ : wavenumber) and their Fourier-transformed (FT) spectra are shown in Figure 9a-d. The EXAFS spectrum of $\mathrm{T}_{1}$ was measured at 100 ps after photoexcitation in which the movement of the ligands subsided. In the 
FT spectra of both $\mathrm{S}_{0}$ (Figure 9b) and $\mathrm{T}_{1}$ (Figure 9d), a dominant contribution at $\sim 1.0-2.3 \AA$ is attributed to the first nearest neighbor $(\mathrm{NN})$ of the Re-C in CO, the Re-N in dmb, and the Re-P in $\mathrm{PPh}_{3}$. The feature appearing at $\sim 2.3-3.2 \AA$ results from contributions from the second $\mathrm{NN}$ of the $\mathrm{Re}-\mathrm{C}$ in dmb and the Re-O in CO, and the multiple scatterings in dmb and CO. To investigate the local molecular structures around the Re atom in more detail, curve-fitting analysis was performed. The $\mathrm{R}$ range employed in the curve-fitting analysis was $\Delta \mathrm{R} \approx 1-3.2 \AA$. Fitting results are shown in Figure 9 as dotted lines. The bond lengths of the first NN obtained from the EXAFS analysis are listed in Table 1 together with those obtained by the DFT calculations from the TR-IR analysis. These values are in good agreement with each other. Also, the values for $\mathrm{S}_{0}$ are very close to those in a single crystal obtained by X-ray diffraction analysis [6]. These results strongly indicate that the optimized geometries by DFT calculations are highly reliable.

The differences in bond length between $S_{0}$ and $T_{1}$ are qualitatively explained by the fact that $\mathrm{T}_{1}$ is the MLCT state. Charge transfer from Re to dmb decreases the charge on Re and increases that on $\mathrm{dmb}$. The bonds between Re and $\mathrm{CO}$ and between Re and dmb have $\mathrm{d}-\pi$ interaction as well as coordination bond whereas that between Re and a phosphine ligand has only coordination bond. A decrease in charge on Re decreases the $\mathrm{d}-\pi$ interaction in Re-C bond between Re and CO (a decrease in $\pi$-back donation) and increases the bond length between Re and $\mathrm{C}$. The bond between Re and dmb undergoes an increase in charge on dmb in addition to a decrease in charge on Re; thus, the $\mathrm{d}-\pi$ interaction somewhat increases and the bond lengths between $\mathrm{Re}$ and $\mathrm{N}$ increase. In contrast, the bond length between Re and P increase by a decrease in charge on Re because the bond between Re and a phosphine ligand has no $d-\pi$ interaction.

Table 1. Bond lengths and mean-square displacements $\sigma^{2}$ in $S_{0}$ and $T_{1}$ obtained by the EXAFS fitting in $(3,3)$, and calculated values obtained by the DFT calculations.

\begin{tabular}{|c|c|c|c|c|c|c|}
\hline \multirow[t]{3}{*}{ bond } & \multicolumn{3}{|c|}{$\mathrm{S}_{0}$} & \multicolumn{3}{|c|}{$\mathrm{T}_{1}$} \\
\hline & \multicolumn{2}{|c|}{ length $(\AA)$} & \multirow{2}{*}{$\frac{\sigma^{2}\left(\AA^{2}\right)}{\text { EXAFS }}$} & \multicolumn{2}{|c|}{ length $(\AA)$} & \multirow{2}{*}{$\begin{array}{l}\sigma^{2}\left(\AA^{2}\right) \\
\text { EXAFS }\end{array}$} \\
\hline & calc. & EXAFS & & calc. & EXAFS & \\
\hline $\mathrm{Re}-\mathrm{C} 1$ & 1.89 & $1.86(2)$ & $0.008(2)$ & 1.96 & $1.95(2)$ & $0.016(13)$ \\
\hline $\mathrm{Re}-\mathrm{C} 2$ & 1.89 & $1.86(2)$ & $0.008(2)$ & 1.90 & $1.89(2)$ & $0.016(13)$ \\
\hline $\mathrm{Re}-\mathrm{P} 1$ & 2.51 & $2.41(2)$ & $0.004(2)$ & 2.56 & $2.54(2)$ & $0.005(3)$ \\
\hline $\mathrm{Re}-\mathrm{P} 2$ & 2.51 & $2.41(2)$ & $0.004(2)$ & 2.56 & $2.54(2)$ & $0.005(3)$ \\
\hline $\mathrm{Re}-\mathrm{N} 1$ & 2.16 & $2.13(5)$ & $0.004(4)$ & 2.14 & $2.11(2)$ & $0.001(1)$ \\
\hline $\mathrm{Re}-\mathrm{N} 2$ & 2.15 & $2.13(5)$ & $0.004(4)$ & 2.05 & $2.03(2)$ & $0.001(1)$ \\
\hline
\end{tabular}

3.4. NTOs and geometries in $\mathrm{S}_{0}$ and $\mathrm{T}_{1}$ 
The calculated difference spectra between $T_{1}$ and $S_{0}$ are in good agreement with the TR-IR spectra and also the optimized geometries are confirmed by the TR-EXAFS spectra. The optimized geometries in $S_{0}$ and $T_{1}$ are therefore sufficiently reliable for further discussion on molecular orbitals and molecular structures. Figure 10 shows NTOs in $\mathrm{S}_{0}$ and $\mathrm{T}_{1}$ for $\operatorname{Et}(2,2)$. NTOs were calculated by TD-DFT based on the optimized geometries, and they express the variation in charge distribution upon transition [40]. For both $\mathrm{S}_{0}$ and $\mathrm{T}_{1}$ geometries, the highest occupied NTO (HONTO) is distributed mainly at Re and slightly at $\mathrm{CO}$ and dmb, whereas the lowest unoccupied NTO (LUNTO) is distributed mainly at dmb and slightly at CO but little at Re. This variation in orbital distribution indicates that charge at Re transfers to dmb, which agrees well with $\mathrm{T}_{1}$ being assigned to the ${ }^{3} \mathrm{MLCT}$ state. In contrast to $\mathrm{CO}$ and dmb, NTOs are little distributed at $\mathrm{Ph}$ and there is almost no change in orbital distribution between the HONTO and LUNTO, indicating that phenyl groups do not undergo changes in charge distribution. These results explain why the structural change of phenyl groups occurs more slowly than charge transfer from Re to dmb after photoexcitation (described above). The phenyl groups are electronically isolated through the C-P-Re bond, so they are relocated by weak interactions with $\mathrm{dmb}$ and/or $\mathrm{CO}$, which can be regarded as $\pi-\pi$ interactions. This process should take several tens picoseconds using the analogy for the reorientation of surrounding solvent molecules. This mechanism also applies in $(3,3)$ because the HONTO and LUNTO are almost the same as those of $\operatorname{Et}(2,2)$, as shown in Figure 11.

We now compare the optimized geometries between $\mathrm{S}_{0}$ and $\mathrm{T}_{1}$. For $\operatorname{Et}(2,2)$, there are two geometries that have slightly different energies: one geometry has both Et groups on the same side (cis-Et $(2,2)$ in Figure 12) and the other geometry has one Et on either side (trans-Et $(2,2)$ in Figure 13). In these figures, the lower and upper geometries are $S_{0}$ and $T_{1}$, respectively. These geometries correspond to two of the conformers in the previous report [6], and they can be verified by comparing their calculated spectra with TR-IR spectra. The calculated difference spectra of these two geometries are almost the same, as shown in Figure S6. Thus, the predominant geometry in solution cannot readily be determined from the TR-IR spectra. The energy difference between these geometries is very small: $0.0069 \mathrm{eV}$ in $\mathrm{S}_{0}$ and $0.0207 \mathrm{eV}$ in $\mathrm{T}_{1}$, but the potential barrier between the two geometries should be much larger considering the reaction path. According to the previous report [6], the barrier heights are around $10 \mathrm{~kJ} / \mathrm{mol}$, which is much higher than the energy at room temperature $(\sim 2.5 \mathrm{~kJ} / \mathrm{mol})$. Thus, these two geometries are not likely to exchange at room temperature.

Figure 14 shows the optimized geometries in $\mathrm{S}_{0}$ (lower) and $\mathrm{T}_{1}$ (upper) for $(3,3)$. Tables $\mathrm{S} 1$ and S2 summarize structural parameters obtained from the calculations for $\operatorname{Et}(2,2)$ and $(3,3)$, respectively. When the geometrical changes from $\mathrm{S}_{0}$ to $\mathrm{T}_{1}$ are considered, the common features in structural change between $\operatorname{Et}(2,2)$ and $(3,3)$ are the elongation of Re-P bonds $(0.05 \AA$ in 
$\operatorname{Et}(2,2)$ and $0.05 \AA$ in $(3,3))$ and the reduction of P-Re-P angle $\left(-4.6^{\circ}\right.$ in $\operatorname{Et}(2,2)$ and $-8.1^{\circ}$ in $(3,3)$ ). These structural changes should occur immediately after photoexcitation because $\mathrm{P}$ is coordinated directly to $\mathrm{Re}$ and $\mathrm{Re}$ is oxidized by the charge transfer from $\mathrm{Re}$ to dmb upon photoexcitation. Similar structural changes are reportedly observed in the dimer consisting of the same Re complex units [21]. The structures of $\mathrm{CO}$ and $\mathrm{dmb}$ are little changed by the charge transfer because the double or triple bonds in these ligands are stronger than P-Re. The electronic states of these ligands are largely changed by charge transfer because large spectral changes are observed immediately after photoexcitation.

In addition to these common features in structural change between $\operatorname{Et}(2,2)$ and $(3,3)$, the rotations of phenyl groups on the C-P axis are different between $\operatorname{Et}(2,2)$ and $(3,3)$. As shown in Tables S1 and S2, the phenyl groups are rotated by $1^{\circ}$ or $2^{\circ}$ in $\operatorname{Et}(2,2)$ and by $16-27^{\circ}$ in $(3,3)$, with respect to the ground state angles. The intensities of vibrational bands assigned to $\mathrm{Ph}$ increase more slowly than the other bands, so these rotations occur much later after the charge transfer upon photoexcitation. There is little change in charge distribution in the phenyl groups upon photoexcitation, indicating that there is little change in electronic interaction of the phenyl groups with other ligands. Thus, these structural changes occur not by direct change in electronic states by the charge transfer but by indirect change induced by the elongation of Re-P bonds.

\subsection{Correlation between photophysical properties and excited state structures}

According to the report [6], there are two characteristics of photophysical properties in $(3,3)$ compared with $(2,2)$ and other complexes:

(1) The Stokes shift of $(3,3)$ is much smaller than that of $\operatorname{Et}(2,2)$,

(2) The lifetime of $T_{1}$ in $(3,3)$ is much longer than that in $\operatorname{Et}(2,2)$.

Our experiments and calculations revealed the following characteristics in terms of electronic state and molecular geometry:

(i) Charge transfer occurs only in the plane consisting of $\mathrm{Re}, \mathrm{CO}$, and $\mathrm{dmb}$, and has no direct effect on $\mathrm{Ph}$,

(ii) Only $\mathrm{Ph}$ undergoes structural change slowly (25-30 ps) after photoexcitation.

Therefore, phenyl groups in the phosphine ligands show different behavior upon photoexcitation, and this behavior is expected to originate from indirect interactions.

The next question is how these experimental observations explain the difference in photophysical properties. Regarding characteristic (1), the quantum chemical calculations of $T_{1}$, which are well supported by TR-IR and TR-EXAFS, effectively reproduce this characteristic as shown in the energy diagram in Figure 15. This means that the calculations include the origin of this characteristic. Close inspection of the distances among the functional groups in the filling models obtained from the calculations (Figure 16) shows that the phenyl group above dmb in 
$\operatorname{Et}(2,2)$ is more parallel to the $\mathrm{dmb}$ plane than that in $(3,3)$. This is probably because the phenyl groups in $(3,3)$ are so crowded that they are rotated to avoid steric repulsions. This rotation reduces the attractive $\pi-\pi$ interaction between the phenyl group and $\mathrm{dmb}$ in $(3,3)$ and increases the repulsive force among the phenyl groups and $\mathrm{dmb}$. This is why $\mathrm{T}_{1}$ in $(3,3)$ is higher in energy than that in $\operatorname{Et}(2,2)$. Figure 17 shows schematic potentials depicting this situation. The crowded phenyl groups in $(3,3)$ create the shallow potential because of steric repulsion, which makes the phenyl groups readily rotate. The deeper potentials because of the non-crowded phenyl groups in $\operatorname{Et}(2,2)$ restrict the rotation of phenyl groups. This difference in potential also explains why the Stokes shift of $(3,3)$ is smaller than that of $\operatorname{Et}(2,2)$.

Regarding characteristic (2) requires considering how the lifetime of $T_{1}$ is determined in these complexes. In general, a lowest triplet excited state has a very long lifetime of more than a millisecond because of spin forbidden transition. The main relaxation paths to the ground state in these types of metal complexes are direct relaxation from $T_{1}$ to $S_{0}$ potentials unless another excited state exists that is close in energy to $T_{1}$. This type of non-radiative relaxation was studied semiquantitatively by Engleman and Jortner [45]. They derived a simple law named the "energy gap law": The relaxation rate is determined by the overlap between wavefunctions of the excited and ground state potentials at the same energy level (Franck-Condon factor). Roughly speaking, a larger energy gap between two potentials gives a smaller Franck-Condon factor and a smaller relaxation rate if the equilibrium positions of the two potentials are close (Figure 18a). A larger difference in equilibrium position gives a larger Franck-Condon factor and a larger relaxation rate if the energy gap is the same (Figure 18b). However, these simple predictions are not reasonable for a molecule in which there is a large difference in molecular structure between the ground and excited states such as in our complexes. Particularly the assumption described in Ref. [45]: "We assume that the normal modes and their frequencies are the same in the two electronic states except for displacements in the origins of the normal coordinates.", is not valid for a complex that undergoes a large structural change upon photoexcitation such as $(3,3)$. This deviation from the simple model is known as the Duschinsky effect [46, 47]. Nevertheless, quantitative estimation of this effect is difficult for a complicated system such as in our complexes, so here we consider it qualitatively. If the normal coordinate of $\mathrm{T}_{1}$ is largely different from that of $\mathrm{S}_{0}$, the FranckCondon factor becomes small, as shown schematically in Figure 18c. The energy gap law considering this effect can explain characteristic (2). While the structural change between $T_{1}$ and $\mathrm{S}_{0}$ in $(3,3)$ is large, that in $(2,2)$ is small. Thus, the total Franck-Condon factor in $(3,3)$ is smaller than that in $(2,2)$, so the lifetime of $\mathrm{T}_{1}$ in $(3,3)$ becomes longer than that in $(2,2)$. This situation is the same as that for the dimer consisting of the same Re complex units we reported previously [21]. 


\section{Summary}

We investigated the photophysical properties of two phenylphosphine $\operatorname{Re}(\mathrm{I})$ diimine biscarbonyl complexes, one bearing two phenyl groups and one ethyl group, $\operatorname{Et}(2,2)$, and the other baring three phenyl groups, $(3,3)$, in one phenylphosphine ligand. The different photophysical properties of these two complexes cannot be explained solely by the electron accepting ability of the phosphine ligands. Thus, we compared their excited state dynamics using the combination method of TR-IR, TR-EXAFS, and DFT calculations. Their TR-IR spectra are effectively reproduced by the DFT calculations, assuming that the ground and excited states are the lowest singlet $\left(\mathrm{S}_{0}\right)$ and lowest triplet $\left(\mathrm{T}_{1}\right)$ states, respectively. Normal vibrational mode analyses were carried out based on the calculations and comparison to spectra of a deuterated complex. The temporal evolutions of TR-IR spectra indicate that the phenyl groups undergo structural change more slowly (by $\sim 20-30 \mathrm{ps}$ ) than the other ligands ( $<1 \mathrm{ps}$ ). The positions of atoms adjacent to Re were determined by TR-EXAFS, which are consistent with the geometries obtained by the DFT calculations.

The natural transition orbital analysis indicates that photoexcitation induces charge transfer from Re to the diimine ligands but no change in charge at the phenyl groups. From the optimized geometries of $\mathrm{S}_{0}$ and $\mathrm{T}_{1}$, the bond length of Re-P and the angle of P-Re-P are changed to a similar degree in both complexes, while the phenyl groups are largely rotated by $16-21^{\circ}$ only in $(3,3)$. These results indicate that that displacements of phenyl groups occur indirectly via steric interaction among the aromatic groups, phenyl groups, and diimine. We therefore concluded that the triplet excited state in $(3,3)$ being located higher in energy than that in $\operatorname{Et}(2,2)$ originates from the steric instability; this is because of the congestion of the phenyl groups in $(3,3)$. The longer lifetime of $(3,3)$ is attributed to the reduced non-radiative relaxation from the smaller overlap of wavefunctions between $S_{0}$ and $T_{1}$ because of the large displacements of phenyl groups, which is a special case of the energy gap law. This correlation between photophysical properties and structural dynamics indicates that photophysical properties in metal complexes can be controlled by carefully placing ligands while considering their steric interactions. In addition, our study shows that the combination method of TR-IR and TR-EXAFS makes it possible for us to explore photophysical and photochemical properties in metal complexes in terms of excited-state structure.

\section{Acknowledgements}

This work was supported by JSPS KAKENHI Grant Numbers JP17H06375, JP17H06438, JP17H06372, JP17H06141, JP18H05170, JP19H05782, JP20H05106 and JST CREST Grant Number JPMJCR13L1. The computations were performed at the Research Institute for Information Technology (Kyushu University, Japan). 


\section{Supporting Information.}

Selected normal vibrational modes of $\operatorname{Et}(2,2)$ and $(3,3)$, comparison of TR-IR spectra of $(3,3)$ and (3D,3D), FT-IR and TR-IR spectra of cis- and trans-Et(2,2), selected bond lengths and dihedral angles in the optimized geometries of $\operatorname{Et}(2,2)$ and $(3,3)$, details of EXAFS analysis.

\section{References}

(1) Yamazaki, Y.; Takeda, H.; Ishitani, O. Photocatalytic reduction of $\mathrm{CO}_{2}$ using metal complexes, J. Photochem. Photobio. C: Photochem. Rev. 2015, 25, 106-137.

(2) Ishitani, O.; George, M. W.; Ibusuki, T.; Johnson, F. P. A.; Koike, K.; Nozaki, K.; Pac, C.; Turner, J. J.; Westwell, J. R. Photophysical Behavior of New $\mathrm{CO}_{2}$ Reduction Catalyst, $\operatorname{Re}(\mathrm{CO})_{2}(\mathrm{bpy})\left\{\mathrm{P}(\mathrm{OEt})_{3}\right\}_{2}{ }^{+}$, Inorg. Chem. 1994, 33, 4712-4717.

(3) Koike, K.; Tanabe, J.; Toyama, S.; Tsubaki, H.; Sakamoto, K.; Westwell, J. R.; Johnson, F. P. A.; Hori, H.; Saitoh, H.; Ishitani, O. New Synthesis Routes to Biscarbonylbipyridinerhenium(I) Complexes cis, trans- $\left[\mathrm{Re}\left(\mathrm{X}_{2}-\text { bpy }\right)(\mathrm{CO})_{2}\left(\mathrm{PR}_{3}\right)(\mathrm{Y})\right]^{\mathrm{n}+} \quad\left(\mathrm{X}_{2}\right.$ bpy $=4,4^{\prime}-\mathrm{X}_{2}-2,2^{\prime}$-bipyridine $)$ via photochemical Ligand Substitution Reactions, and Their Photophysical and Electrochemical Properties, Inorg. Chem. 2000, 39, 2777-2783.

(4) Tsubaki, H.; Sekine. A.; Ohashi, Y.; Koike, K.; Takeda, H.; Ishitani, O. J. Am. Chem. Soc. 2005, 127, 15544-15555.

(5) Takeda, H.; Koike, K.; Inoue, H.; Ishitani, O. Development of an Efficient Photocatalytic System for $\mathrm{CO}_{2}$ Reduction Using Rhenium(I) Complexes Based on Mechanistic Studies. J. Am. Chem. Soc. 2008, 130, 2023-2031.

(6) Morimoto, T.; Ito, M.; Koike, K.; Kojima, T.; Ozeki, T.; Ishitani, O. Dual Emission from Rhenium(I) Compelxes Induced by and Interligand Aromatic Interaction, Chem. Eur. J. 2012, 18, 3292-3304.

(7) Yamamoto, Y.; Sawa, S.; Funada, Y.; Morimoto, T.; Falkenström, M.; Miyasaka, H.; Shishido, S.; Ozeki, T.; Koike, K.; Ishitani, O. Systematic Synthesis, Isolation, and Photophysical Properties of Linear-Shaped Re(I) Oligomers and Polymers with 2-20 Units. J. Am. Chem. Soc. 2008, 130, 14659-14674.

(8) Yamamoto, Y.; Tamaki, Y.; Yui, T.; Koike, K.; Ishitani, O. New Light-Harvesting Molecular Systems Constructed with a Ru(II) Complex and a Linear-Shaped Re(I) Oligomer. J. Am. Chem. Soc. 2010, 132, 11743-11752.

(9) Morimoto, T.; Nishiura, C.; Tanaka, M.; Rohacova, J.; Nakagawa, Y.; Funada, Y.; Koike, K.; Yamamoto, Y.; Shishido, S.; Kojima, T.; Saeki, T.; Ozeki, T.; Ishitani, O. Ring-Shaped Re(I) Multinuclear Complexes with Unique Photofunctional Properties. J. Am. Chem. Soc. 2013, 135, 
13266-13269.

(10) Asatani, T.; Nakagawa, Y.; Funada, Y.; Swa, S.; Takeda, H.; Morimoto, T.; Koike, K.; Ishitani, O. Ring-Shaped Rhenium(I) Multinuclear Complexes: Improved Synthesis and Photoinduced Multielectron Accumulation, Inorg. Chem. 2014, 53, 7170-7180.

(11) Rohacova, J.; Sekine, A.; Kawano, T.; Tamri, S.; Ishitani, O. Trinuclear nad Tetranuclear $\operatorname{Re}(\mathrm{I})$ Rings Connencted with Phenylene Vinylene, and Ethynylene Chains: Synthesis, Photophysics, and Redox Properties, Inorg. Chem. 2015, 54, 8769-8777.

(12) Rohacova, J.; Ishitani, O. Rhenium(I) trinuclear rings as highly efficient redox photosensitizers for photocatalytic $\mathrm{CO}_{2}$ reduction, Chem. Sci. 2016, 7, 6728-6739

(13) Yamamoto, Y.; Takeda, H.; Yui, T.; Ueda, Y.; Koike, K.; Inagaki, S.; Ishitani, O., Efficient light harvesting via sequential two-step energy accumulation using a Ru-Re5 multinuclear complex incorporated into periodic mesoporous organosilica. Chemical Science 2014, 5, 639-648.

(14) Martinez, C. R.; Iverson, B. L. Rethinking the term "pi-stacking", Chem. Sci. 2012, 3, 2191 2201.

(15) Hunter, C. A.; J. K. M. Sanders, The Nature of $\pi-\pi$ Interactions, J. Am. Chem. Soc. 1990, 112, 5525-5534.

(16) Hunter, C. A. Lawson, K. R.; Perkins, J.; Urch, C. J. Aromatic interactions, J. Chem. Soc., Perkin Trans, 2001, 2, 651-669.

(17) Sinnokrot, M. O.; Valeev, E. F.; Sherrill, C. D. Estimates of the Ab Initio Limit for $\pi-\pi$ Interactions: The Benzene Dimer, J. Am. Chem. Soc. 2002, 124, 10887-10893.

(18) Butler, J. M.; George M. W.; Schoonover, J. R.; Dattelbaum, D. M.; Meyer, T. J. Coord. Chem. Rev. 2007, 251, 492-514.

(19) Fukazawa, N.; Tanaka, T.; Ishikawa, T.; Okimoto, Y.; Koshihara, S.; Yamamoto, T.; Tamura, M.; Kato, R.; Onda, K. Time-Resolved Infrared Vibrational Spectroscopy of the Photoinduced Phase Transition of Pd(dmit) $)_{2}$ Salts Having Different Orders of Phase Transition, J. Phys. Chem. C, 2013, 117, 13187-13196.

(20) Mukuta, T.; Fukazawa, N.; Murata, K.; Inagaki, A.; Akita, M.; Tanaka, S.; Koshihara, S.; Onda, K. Infrared Vibrational Spectroscopy of $\left[\mathrm{Ru}(\mathrm{bpy})_{2}(\mathrm{bpm})\right]^{2+}$ and $\left[\mathrm{Ru}(\mathrm{bpy})_{3}\right]^{2+}$ in the Excited Triplet State. Inorg. Chem. 2014, 53, 2481-2490.

(21) Tanaka, S.; Takahashi, K.; Hirahara, M.; Yagi, M.; Onda, K. Characterization of the excited states of distal- and proximal-[Ru(tpy)(pynp) $\left.\mathrm{OH}_{2}\right]^{2+}$ in aqueous solution using time-resolved infrared spectroscopy. J. Photochem. Photobiol., A 2015, 313, 87-98.

(22) Zimmer, M. ; Rupp, F.; Singer, P.; Walz, F.; Breher, F.; Klopper, W.; Diller, R.; Gerhards, M. Time-resolved IR spectroscopy of a trinuclear palladium complex in solution, Phys. Chem. Chem. Phys. 2015, 17, 14138-14144. 
(23) Mukuta, T.; Tanaka, S.; Inagaki, A.; Koshihara, S.; Onda, K. Direct Observation of the Triplet Metal-Centered State in $\left[\mathrm{Ru}(\mathrm{bpy})_{3}\right]^{2+}$ Using Time-Resolved Infrared Spectroscopy. ChemistrySelect 2016, 1, 2802-2807.

(24) Matsubara, Y.; Okimoto, Y.; Yoshida, T.; Ishikawa, T.; Koshihara, S.; Onda, K. Photoinduced Neutral-to-Ionic Phase Transition in Tetrathiafulvalene- $p$-Chloranil Studied by Time-resolved Vibrational Spectroscopy, J. Phys. Soc. Jpn, 2011, 80, 124711-1 - -5.

(25) Fukazawa, N.; Shimizu, M.; Ishikawa, T.; Okimoto, Y.; Koshihara, S.; Hiramatsu, T.; Nakano, Y.; Yamochi, H.; Saito, G.; Onda, K. Charge and structural dynamics in photoinduced phase transition of (EDO-TTF $)_{2} \mathrm{PF}_{6}$ examined by picosecond time-resolved vibrational spectroscopy, $J$. Phys. Chem. C, 2012, 116, 5892-5899.

(26) Hada, M.; Saito, S.; Tanaka, S.; Sato, R.; Yoshimura, M.; Mouri, K.; Matsuo, K.; Yamaguchi, S.; Hara, M.; Hayashi, Y.; Shigeta, Y.; Onda, K.; Miller, R. J. D. Structural Monitoring of the Onset of Excited-State Aromaticity, J. Am. Chem. Soc. 2017, 139, 15792-15800.

(27) Saigo, M.; Miyata, K.; Tanaka, S.; Nakanotani, H.; Adachi, C.; Onda, K. Suppression of Structural Change upon $\mathrm{S}_{1}-\mathrm{T}_{1}$ Conversion Assists Thermally Activated Delayed Fluorescence Process in Carbazole-Benzonitrile Derivatives, J. Phys. Chem. Lett. 2019, 10, 2475-2480.

(28) Sayers, D. E.; Stern, E. A.; Lytle, F. W., New technique for investigating noncrystalline structures: Fourier analysis of the extended x-ray-absorption fine structure. Physical review letters 1971, 27 (18), 1204.

(29) Lytle, F. W., The EXAFS family tree: a personal history of the development of extended Xray absorption fine structure. Journal of Synchrotron Radiation 1999, 6 (3), 123-134.

(30) Gawelda, W.; Pham, V.-T.; Benfatto, M.; Zaushitsyn, Y.; Kaiser, M.; Grolimund, D.; Johnson, S. L.; Abela, R.; Hauser, A.; Bressler, C., Structural determination of a short-lived excited iron (II) complex by picosecond x-ray absorption spectroscopy. Physical review letters 2007, 98 (5), 057401.

(31) Bressler, C.; Chergui, M., Molecular structural dynamics probed by ultrafast X-ray absorption spectroscopy. Annual review of physical chemistry 2010, 61, 263-282.

(32) Nozawa, S.; Sato, T.; Chollet, M.; Ichiyanagi, K.; Tomita, A.; Fujii, H.; Adachi, S.; Koshihara, S., Direct probing of spin state dynamics coupled with electronic and structural modifications by picosecond time-resolved XAFS. Journal of the American Chemical Society 2010, 132 (1), 6163.

(33) Penfold, T. J.; Milne, C. J.; Chergui, M., Recent advances in ultrafast x-ray absorption spectroscopy of solutions. Adv. Chem. Phys 2013, 153, 1-41.

(34) El Nahhas, A.; Van Der Veen, R. M.; Penfold, T. J.; Pham, V. T.; Lima, F. A.; Abela, R.; Blanco-Rodriguez, A. M.; Zalis, S.; Vlcek, A.; Tavernelli, I., X-ray absorption spectroscopy of ground and excited rhenium-carbonyl-diimine complexes: Evidence for a two-center electron 
transfer. The Journal of Physical Chemistry A 2013, 117 (2), 361-369.

(35) Milne, C. J.; Penfold, T. J.; Chergui, M., Recent experimental and theoretical developments in time-resolved X-ray spectroscopies. Coordination Chemistry Reviews 2014, 277, 44-68.

(36) Chergui, M., Ultrafast photophysics of transition metal complexes. Accounts of Chemical Research 2015, 48 (3), 801-808.

(37) Nozawa, S.; Adachi, S. i.; Takahashi, J. i.; Tazaki, R.; Guérin, L.; Daimon, M.; Tomita, A.; Sato, T.; Chollet, M.; Collet, E., Developing 100 ps-resolved X-ray structural analysis capabilities on beamline NW14A at the Photon Factory Advanced Ring. Journal of synchrotron radiation 2007, 14 (4), 313-319.

(38) Gaussian 16, Revision A.03, M. J. Frisch, G. W. Trucks, H. B. Schlegel, G. E. Scuseria, M. A. Robb, J. R. Cheeseman, G. Scalmani, V. Barone, G. A. Petersson, H. Nakatsuji, X. Li, M. Caricato, A. V. Marenich, J. Bloino, B. G. Janesko, R. Gomperts, B. Mennucci, H. P. Hratchian, J. V. Ortiz, A. F. Izmaylov, J. L. Sonnenberg, D. Williams-Young, F. Ding, F. Lipparini, F. Egidi, J. Goings, B. Peng, A. Petrone, T. Henderson, D. Ranasinghe, V. G. Zakrzewski, J. Gao, N. Rega, G. Zheng, W. Liang, M. Hada, M. Ehara, K. Toyota, R. Fukuda, J. Hasegawa, M. Ishida, T. Nakajima, Y. Honda, O. Kitao, H. Nakai, T. Vreven, K. Throssell, J. A. Montgomery, Jr., J. E. Peralta, F. Ogliaro, M. J. Bearpark, J. J. Heyd, E. N. Brothers, K. N. Kudin, V. N. Staroverov, T. A. Keith, R. Kobayashi, J. Normand, K. Raghavachari, A. P. Rendell, J. C. Burant, S. S. Iyengar, J. Tomasi, M. Cossi, J. M. Millam, M. Klene, C. Adamo, R. Cammi, J. W. Ochterski, R. L. Martin, K. Morokuma, O. Farkas, J. B. Foresman, and D. J. Fox, Gaussian, Inc., Wallingford CT, 2016.

(39) Tanaka, S.; Matsubara, Y.; Asatani, T.; Morimoto, T.; Ishitani, O.; Onda, K. Structural deformation of a ring-shaped $\operatorname{Re}(\mathrm{I})$ diimine dinuclear complex in the excited state. Chem. Phys. Lett. 2016, 662, 120-126.

(40) Martin, R. L. Natural transition orbitals, J. Chem. Phys. 2003, 118, 4775-4777.

(41) Sato, S.; Matubara, Y.; Koike, K.; Falkenström, M.; Katayama, T.; Ishibashi, Y.; Miyasaka, H.; Taniguchi, S.; Chosrowjan, H.; Mataga, N.; Fukazawa, N.; Koshihara, S.; Onda, K.; Ishitani, O. Photochemistry of fac-[Re(bpy)(CO) $\left.)_{3} \mathrm{Cl}\right]$. Chem. - Eur. J. 2012, 18, 15722-15734.

(42) Liard, D. J.; Busby, M.; Matousek, P.; Towrie, M.; Vlček, A. Picosecond Relaxation of ${ }^{3}$ MLCT Excited States of $\left[\operatorname{Re}(\operatorname{Etpy})(\mathrm{CO})_{3}(\mathrm{dmb})\right]^{+}$and $\left[\operatorname{Re}(\mathrm{Cl})(\mathrm{CO})_{3}(\right.$ bpy $\left.)\right]$ as Revealed by TimeResolved Resonance Raman, UV-vis, and IR Absorption Spectroscopy. J. Phys. Chem. A 2004, 108, 2363-2369.

(43) Li, G.; Parimal, K.; Vyas, S.; Hadad, C. M.; Flood, A. H.; Glusac, K. D. Pinpointing the Extent of Electronic Delocalization in the Re(I)-to-Tetrazine Charge-Separated Excited State Using Time-Resolved Infrared Spectroscopy. J. Am. Chem. Soc. 2009, 131, 11656-11657.

(44) Dattelbaum, D. M.; Omberg, K. M.; Schoonover, J. R.; Martin, R. L.; Meyer, T. J. Application 
of Time-Resolved Infrared Spectroscopy to Electronic Structure in Metal-to-Ligand ChargeTransfer Excited States. Inorg. Chem. 2002, 41, 6071-6079.

(45) Engleman, R.; Jortner, J. The energy gap law for radiationless transitions in large molecules, Mol. Phys. 1970, 18, 145-164.

(46) Small, G. J. Herzberg-Teller Vibronic Coupling and the Duschinsky Effect, J. Chem. Phys. 1971, 54, 3300-3306.

(47) Mebel, A. M.; Hayashi, M.; Liang, K. K.; Lin, S. H. Ab Initio Calculations of Vibronic Spectra and Dynamics for Small Polyatomic Molecules: Role of Duschinsky Effect, J. Phys. Chem. A, 1999, 103, 10674-10690. 


\section{Figure captions}

Fig. 1. Molecular structures of (a) cis, trans-[Re(dmb)(CO) $\left.)_{2}\left(\mathrm{PPh}_{2} \mathrm{Et}\right)_{2}\right]^{+}(\operatorname{Et}(2,2))$, (b) cis, trans$\left[\operatorname{Re}(\mathrm{dmb})(\mathrm{CO})_{2}\left(\mathrm{PPh}_{3}\right)_{2}\right]^{+}((3,3))$, and $(\mathrm{c})$ deuterated $(3,3)((3 \mathrm{D}, 3 \mathrm{D}))$.

Fig. 2. Steady-state observed and calculated spectra of $\operatorname{Et}(2,2)$ and $(3,3)$ : (a) FT-IR spectra and (b) calculated IR spectra of the $S_{0}$ state of Et(2,2), (c) FT-IR spectra and (d) calculated IR spectra of the $\mathrm{S}_{0}$ state of $(3,3)$. The colors indicate the ligand on which the vibrations are predominantly localized: blue $=\mathrm{CO}$, green $=\mathrm{dmb}$, and red $=\mathrm{Ph}$.

Fig. 3. Time-resolved observed and calculated spectra of $\operatorname{Et}(2,2)$ and $(3,3)$ : (a) TR-IR spectra and (b) calculated difference IR spectra between the $\mathrm{T}_{1}$ and $\mathrm{S}_{0}$ states of Et(2,2), (c) TR-IR spectra and (d) calculated difference IR spectra of the $\mathrm{T}_{1}$ and $\mathrm{S}_{0}$ states of $(3,3)$. The colors indicate the ligand on which the vibrations are predominantly localized: blue $=\mathrm{CO}$, green $=\mathrm{dmb}$, and red $=\mathrm{Ph}$.

Fig. 4. (a) TR-IR spectra and (b) calculated difference IR spectra between the $T_{1}$ and $S_{0}$ states of (3D,3D). (c) Comparison of the TR-IR spectra of (3,3), black line, and (3D,3D), red line.

Fig. 5. Temporal evolutions of the TR-IR spectra of (a) $\operatorname{Et}(2,2)$ and (b) $(3,3)$ in the CO stretching vibrational mode region.

Fig. 6. Temporal evolutions of the TR-IR spectra of (a) $\operatorname{Et}(2,2)$ and (b) $(3,3)$ in the region in which dmb vibrational modes are located.

Fig. 7. Temporal evolutions of the TR-IR spectra of (a) $\operatorname{Et}(2,2)$ and $(b)(3,3)$ in the region in which $\mathrm{Ph}$ and $\mathrm{dmb}$ vibrational modes are located.

Fig. 8. Temporal profiles of the intensity estimated by fitting using a Gaussian function for bands assigned to $\mathrm{CO}$ (blue) and $\mathrm{dmb}$ (green), and of the absorbance change ( $\Delta$ abs.) at the position mainly assigned to $\mathrm{Ph}$ (red) in (a) $\operatorname{Et}(2,2)$ and (b) $(3,3)$. See the text for details of the estimations.

Fig. 9. EXAFS of the $\mathrm{S}_{0}(\mathrm{a})$ and $\mathrm{T}_{1}$ (c) states, and Fourier-transformed (FT) EXAFS of the $\mathrm{S}_{0}(\mathrm{~b})$ and $T_{1}$ (d) states of $(3,3)$. The EXAFS of the $T_{1}$ state is derived by eq. (1) in the supporting information, with the EXAFS of the $\mathrm{S}_{0}$ state shown in (a), 1.4\% of the fraction for $\mathrm{T}_{1}$ at $\tau=100$ ps, and the transient EXAFS difference at 100 ps. In (a) and (c), the windows are for the Fourier transformation, and EXAFS data and their fitting results are indicated by the blue lines and orange 
dotted lines, respectively. In (b) and (d), the windows are for the EXAFS fitting analysis. The experimental data of the real and imaginary parts of FT EXAFS are indicated by the blue and light-blue lines, respectively. The fitting results for the real and imaginary parts of FT EXAFS are indicated by the blue and light-blue dotted lines, respectively.

Fig. 10. Natural transition orbitals for the $\mathrm{S}_{0}$ and $\mathrm{T}_{1}$ states of $\operatorname{Et}(2,2)$.

Fig. 11. Natural transition orbitals for the $S_{0}$ and $T_{1}$ states of $(3,3)$.

Fig. 12. Optimized geometries of the $\mathrm{S}_{0}$ and $\mathrm{T}_{1}$ states of $c i s-\mathrm{Et}(2,2)$.

Fig. 13. Optimized geometries of the $\mathrm{S}_{0}$ and $\mathrm{T}_{1}$ states of trans-Et(2,2).

Fig. 14. Optimized geometries of the $\mathrm{S}_{0}$ and $\mathrm{T}_{1}$ states of $(3,3)$.

Fig. 15. Energy diagrams of the $S_{0}, S_{1}$, and $T_{1}$ states of $\operatorname{Et}(2,2)$ and $(3,3)$. Observed values were estimated from the absorption and emission wavelengths. Calculated emission values were obtained by TD-DFT calculations using the optimized geometry of the $\mathrm{T}_{1}$ state. Calculated Stokes shift values are difference between the observed absorption and calculated emission values.

Fig. 16. Filling models of optimized geometries of $\operatorname{Et}(2,2)$ and $(3,3)$.

Fig. 17. Schematic potentials of $(3,3)$ with crowded phenyl groups and $E t(2,2)$ with non-crowded phenyl groups.

Fig. 18. Schematic Frank-Condon factors in three different configurations between the ground and excited states: (a) same equilibrium position in the same normal coordinate, (b) different equilibrium position in the same normal coordinate, (c) two potentials located in different normal coordinates. 
Figure 1.

(a)

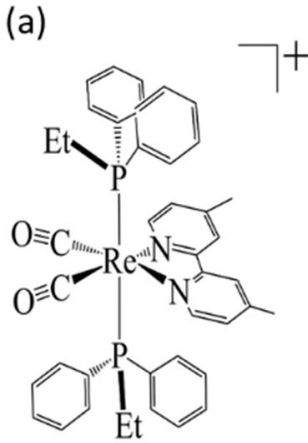

$\mathrm{Et}(2,2)$ (b)

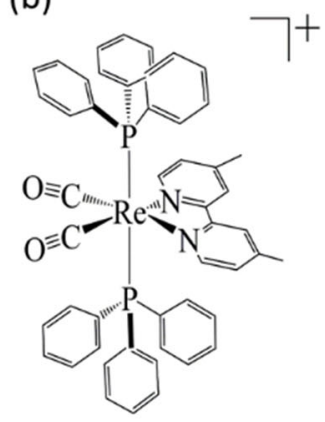

$(3,3)$ (c)

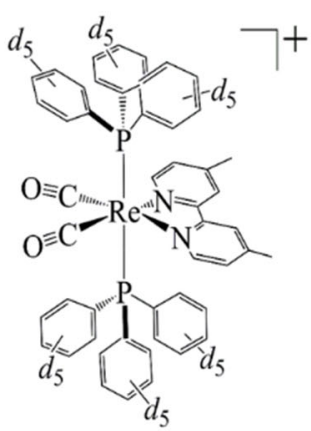

(3D,3D) 
Figure 2.

$\mathrm{Et}(2,2)$

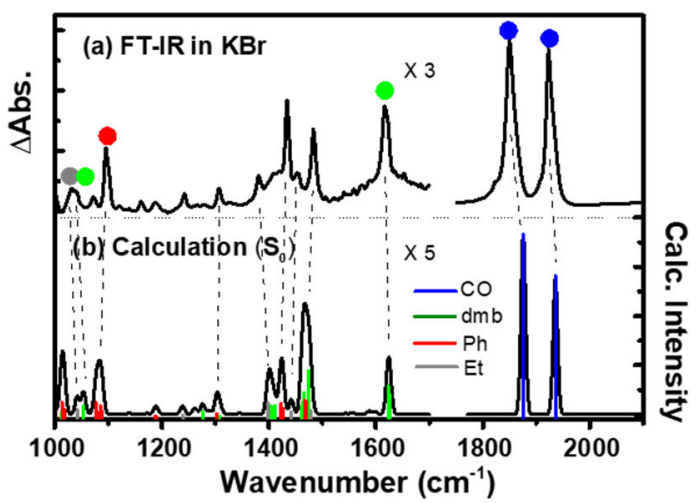

$(3,3)$

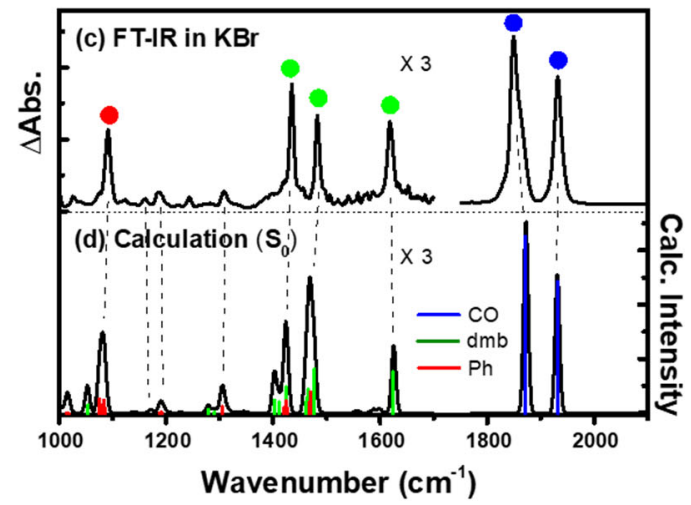


Figure 3.

Et $(2,2)$

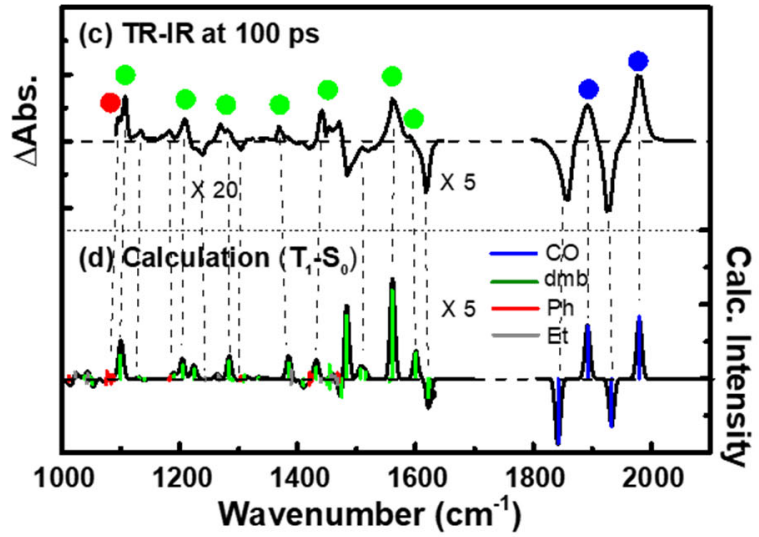

$(3,3)$

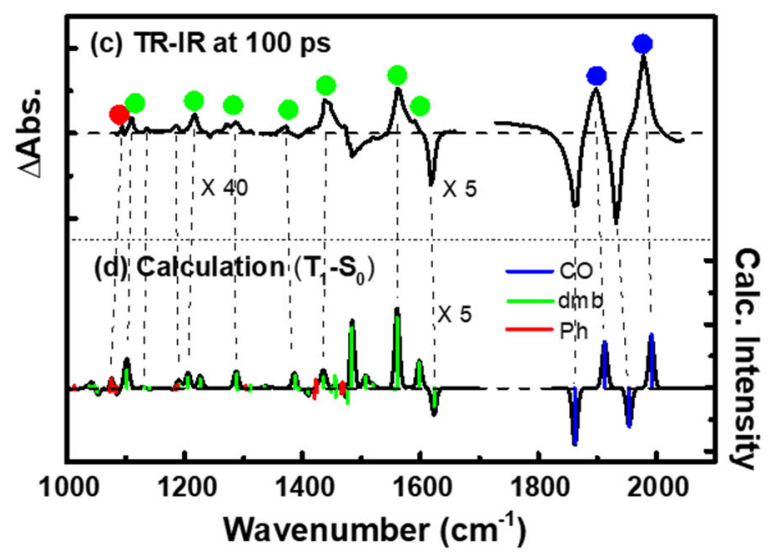


Figure 4.
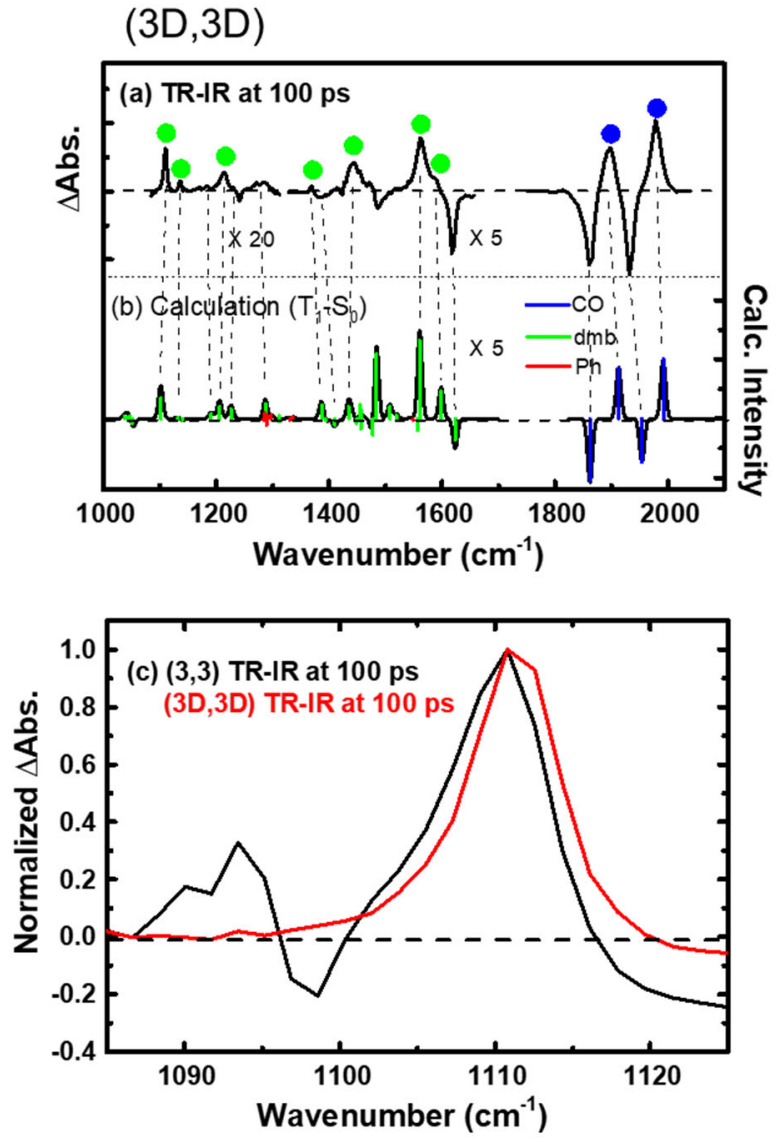
Figure 5.

(a) $\mathrm{Et}(2,2)$

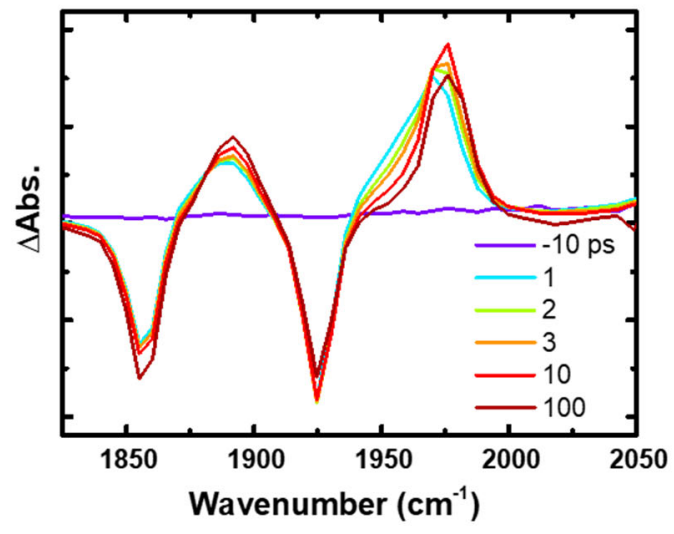

(b) $(3,3)$

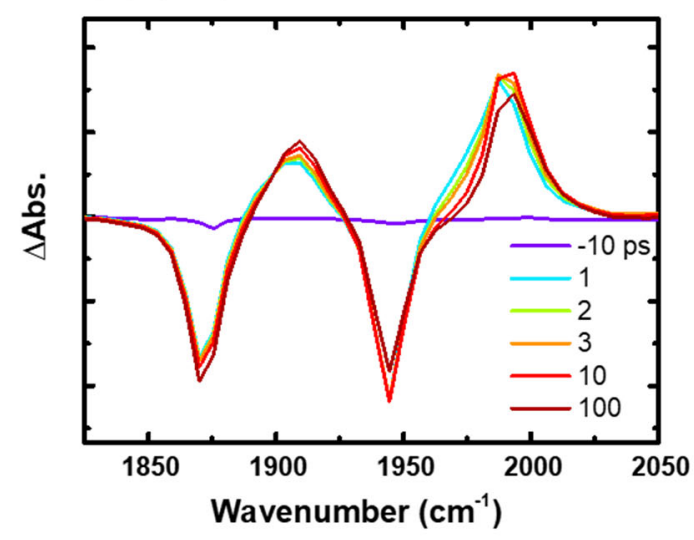


Figure 6.

(a) $\mathrm{Et}(2,2)$

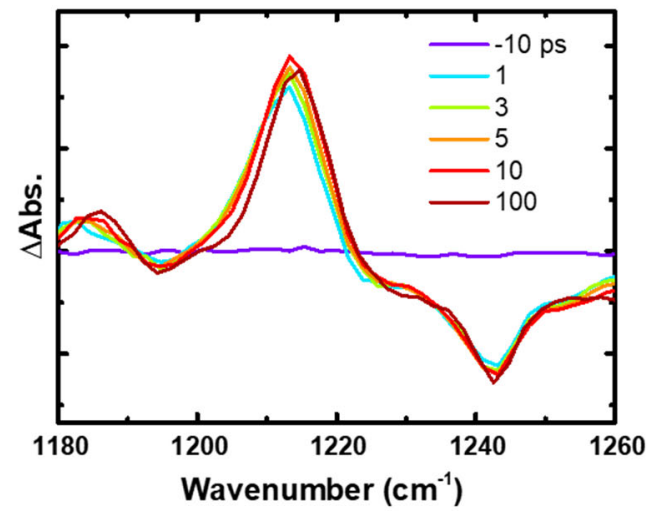

(b) $(3,3)$

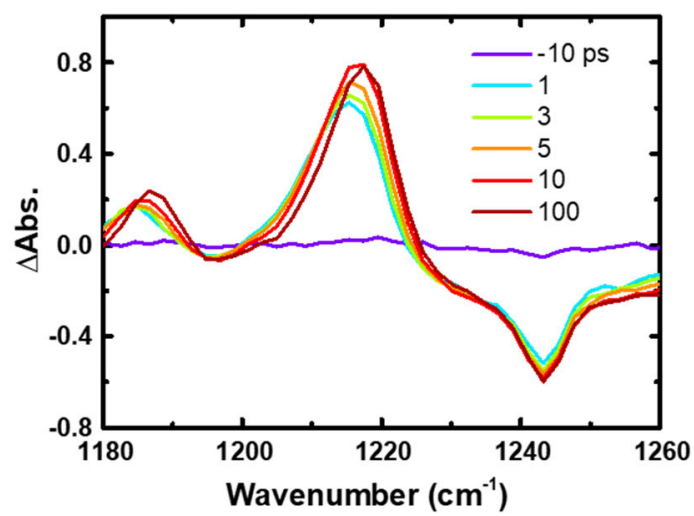


Figure 7.

(a) $\mathrm{Et}(2,2)$

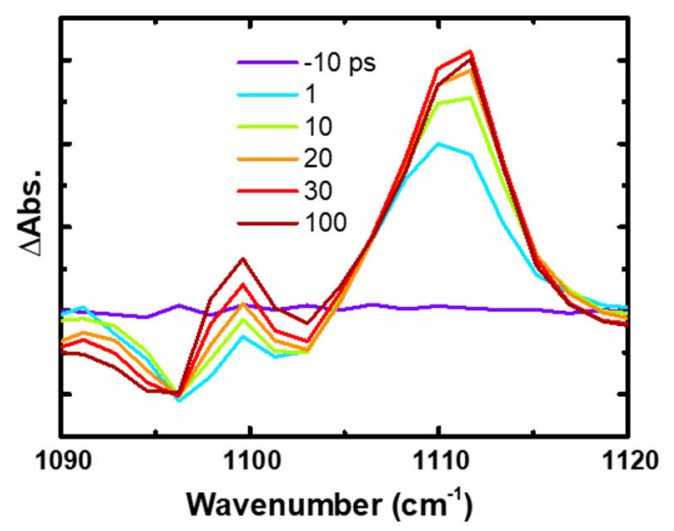

(b) $(3,3)$

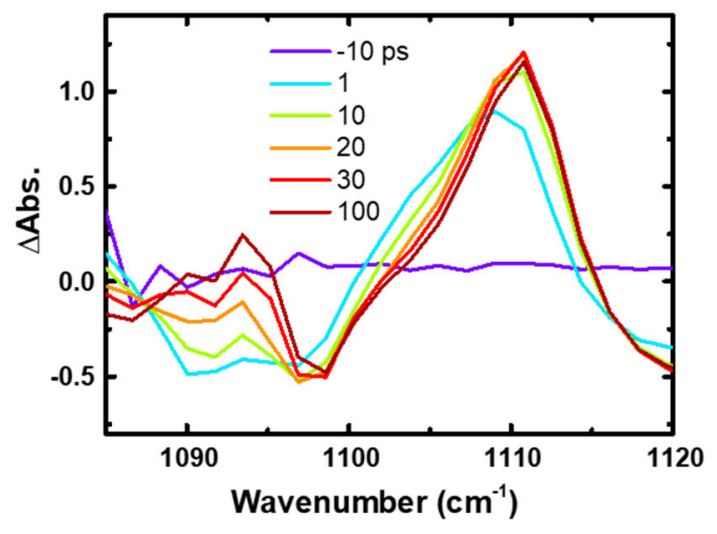


Figure 8.

(a) $\mathrm{Et}(2,2)$

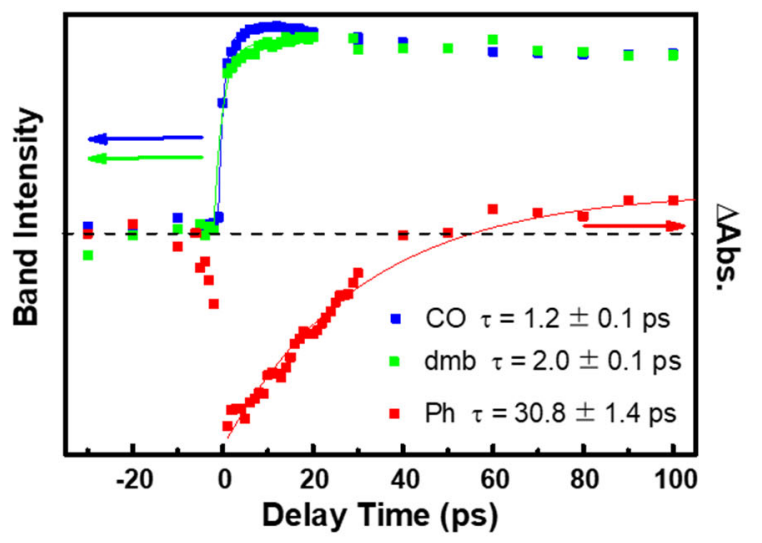

(b) $(3,3)$

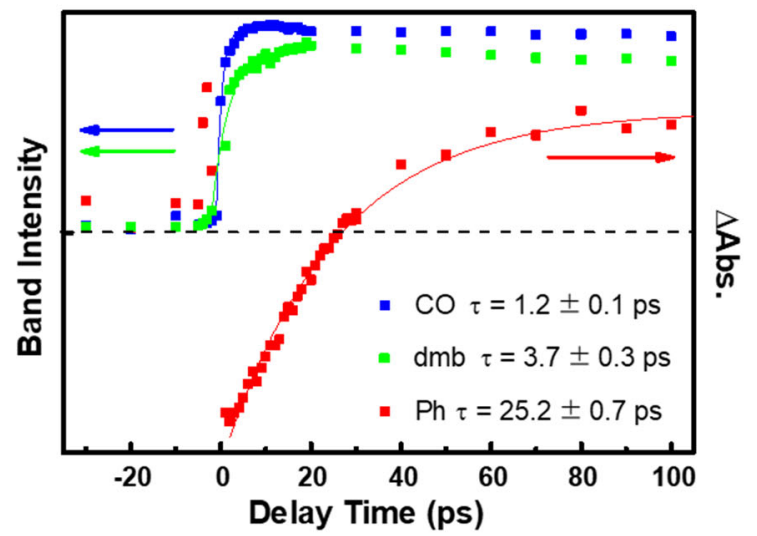


Figure 9.
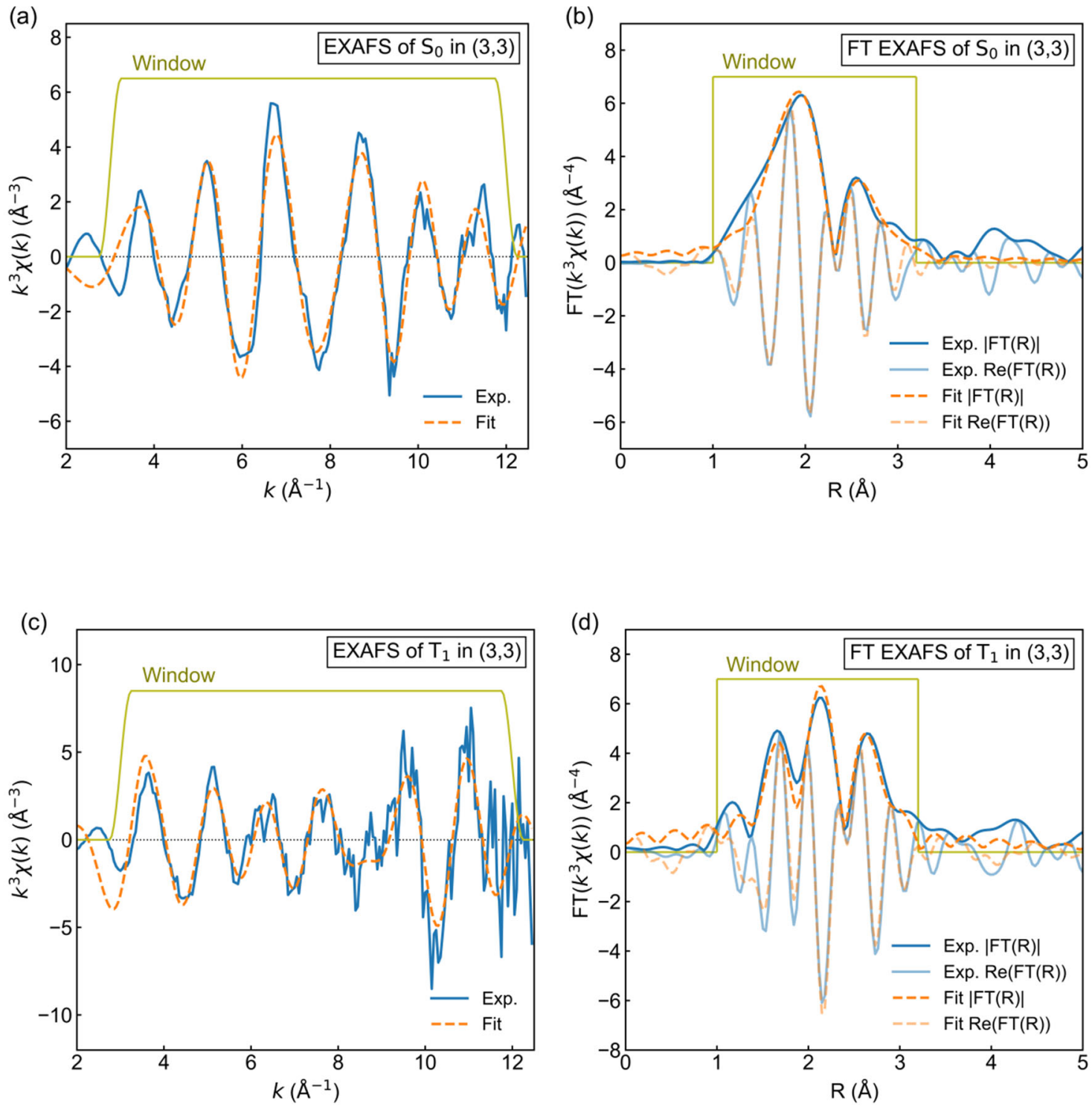
Figure 10.

Et(2,2)
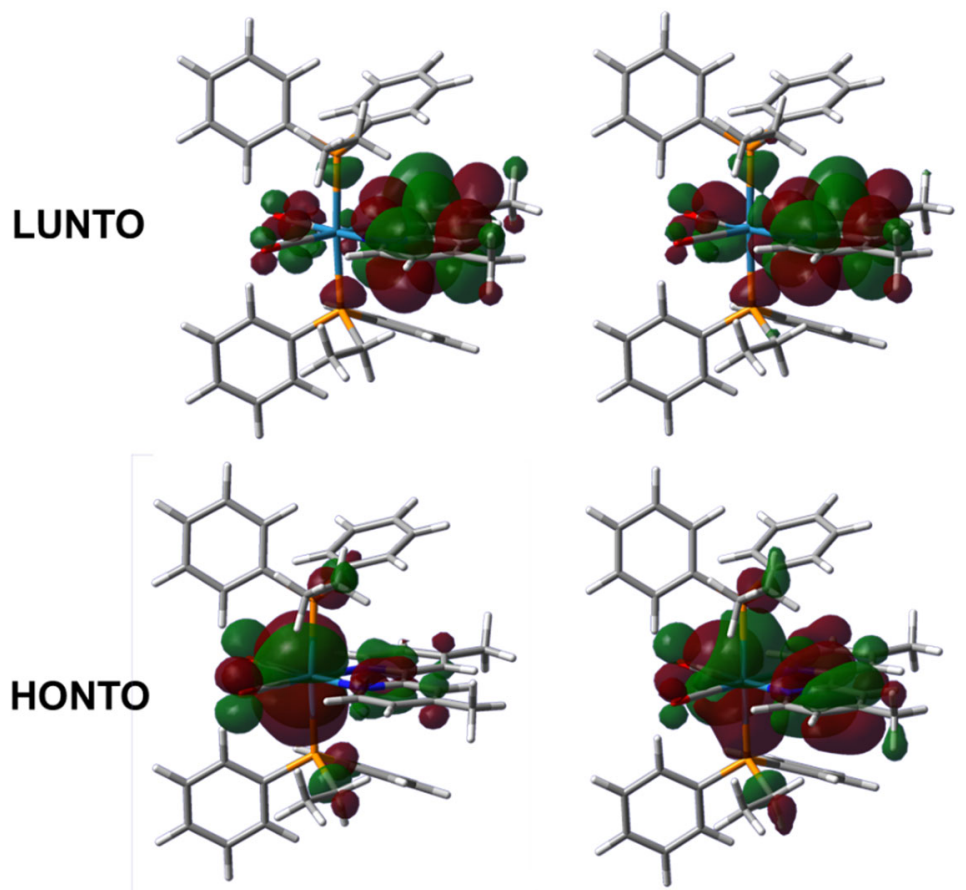

Ground state $\left(\mathbf{S}_{0}\right)$

Excited state $\left(T_{1}\right)$ 
Figure 11.

$(3,3)$

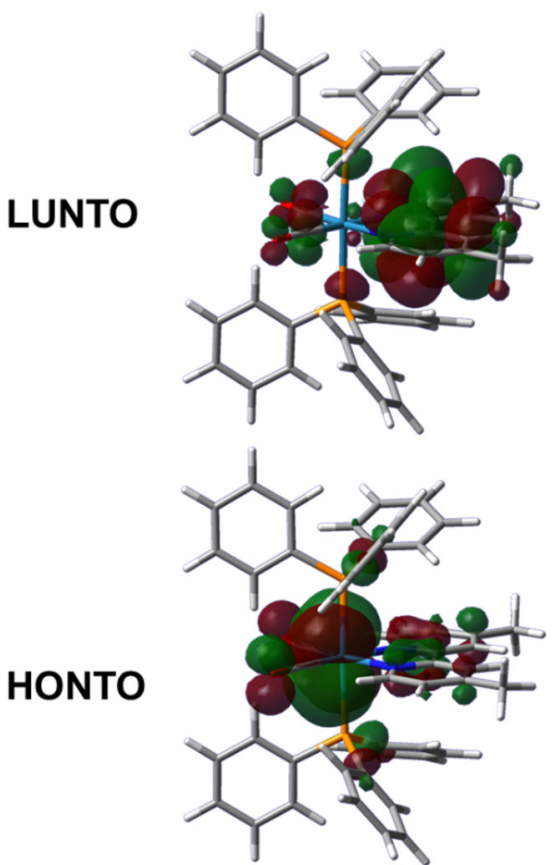

Ground state $\left(\mathbf{S}_{0}\right)$
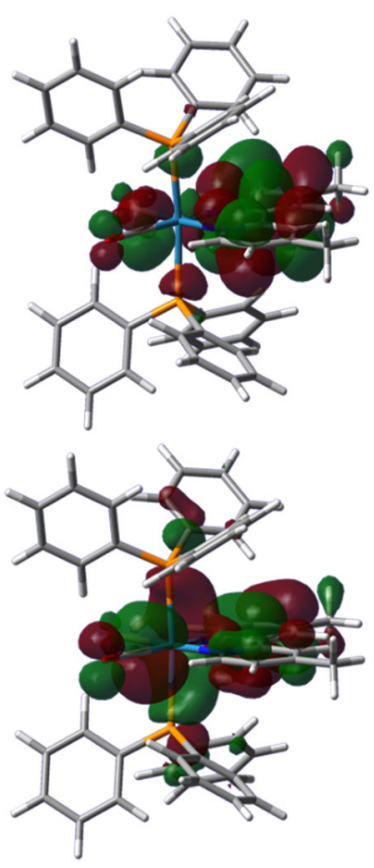

Excited state $\left(T_{1}\right)$ 
Figure 12.

cis-Et(2,2)
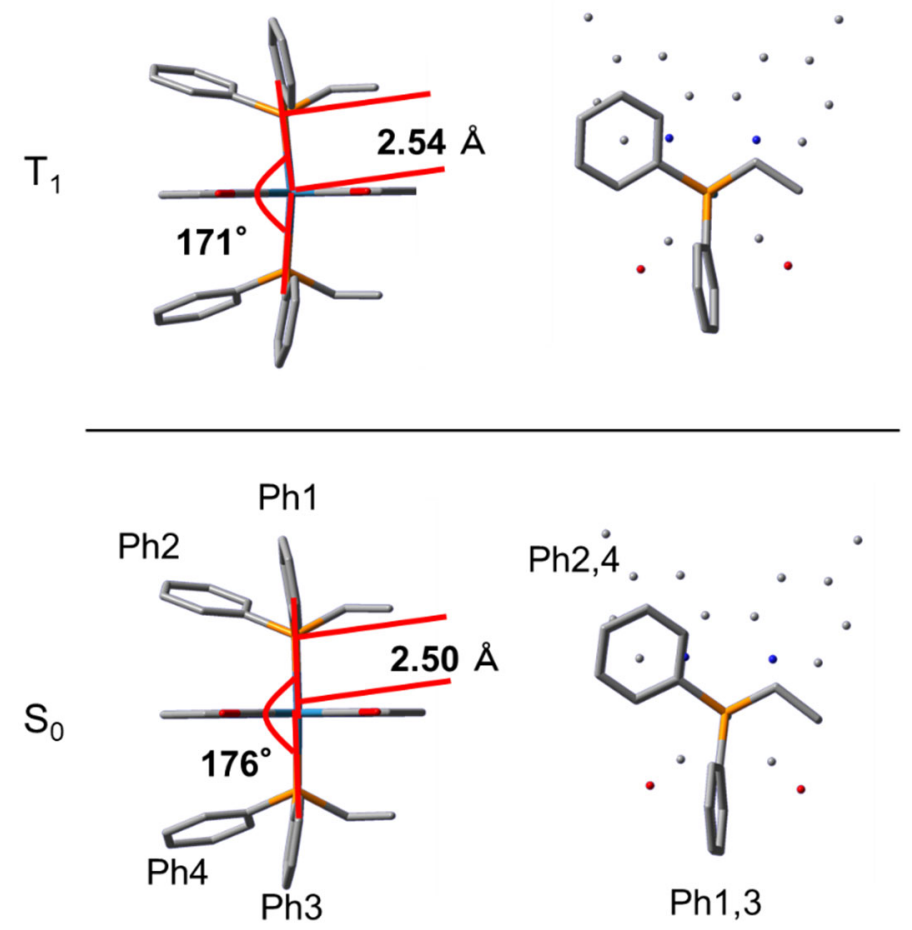
Figure 13.

trans-Et(2,2)
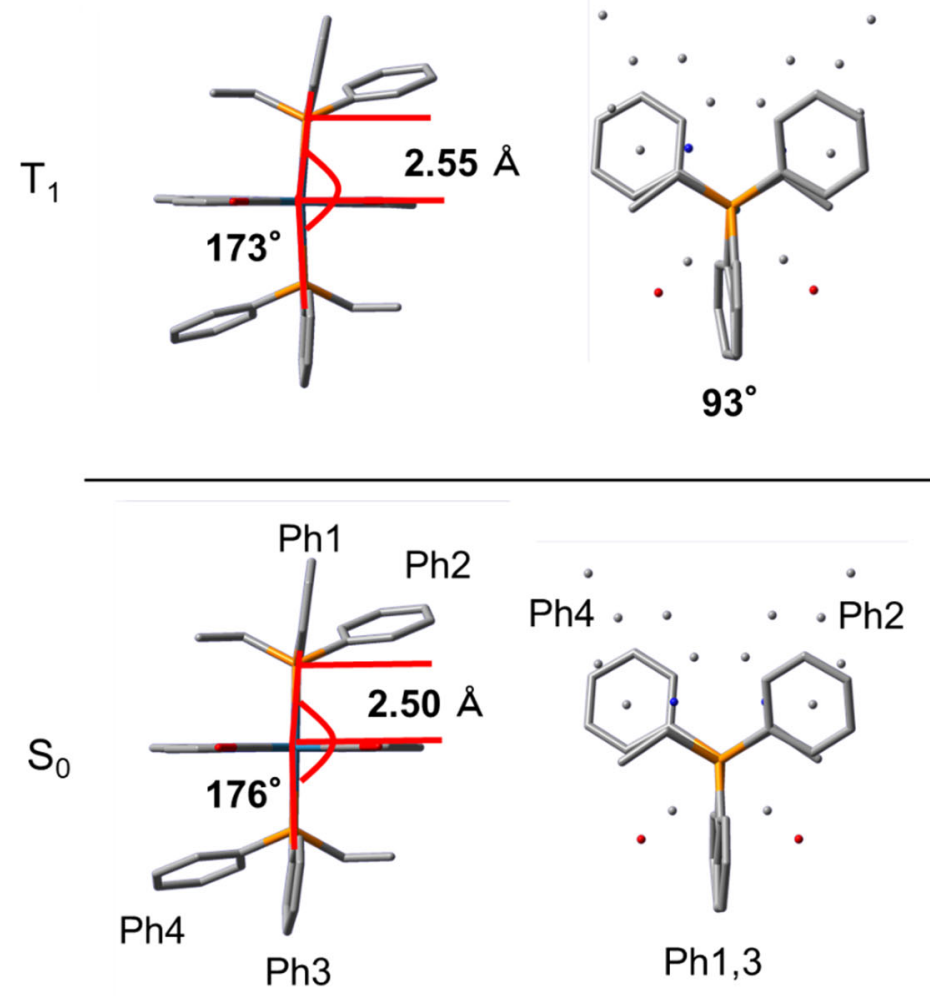
Figure 14.

$(3,3)$
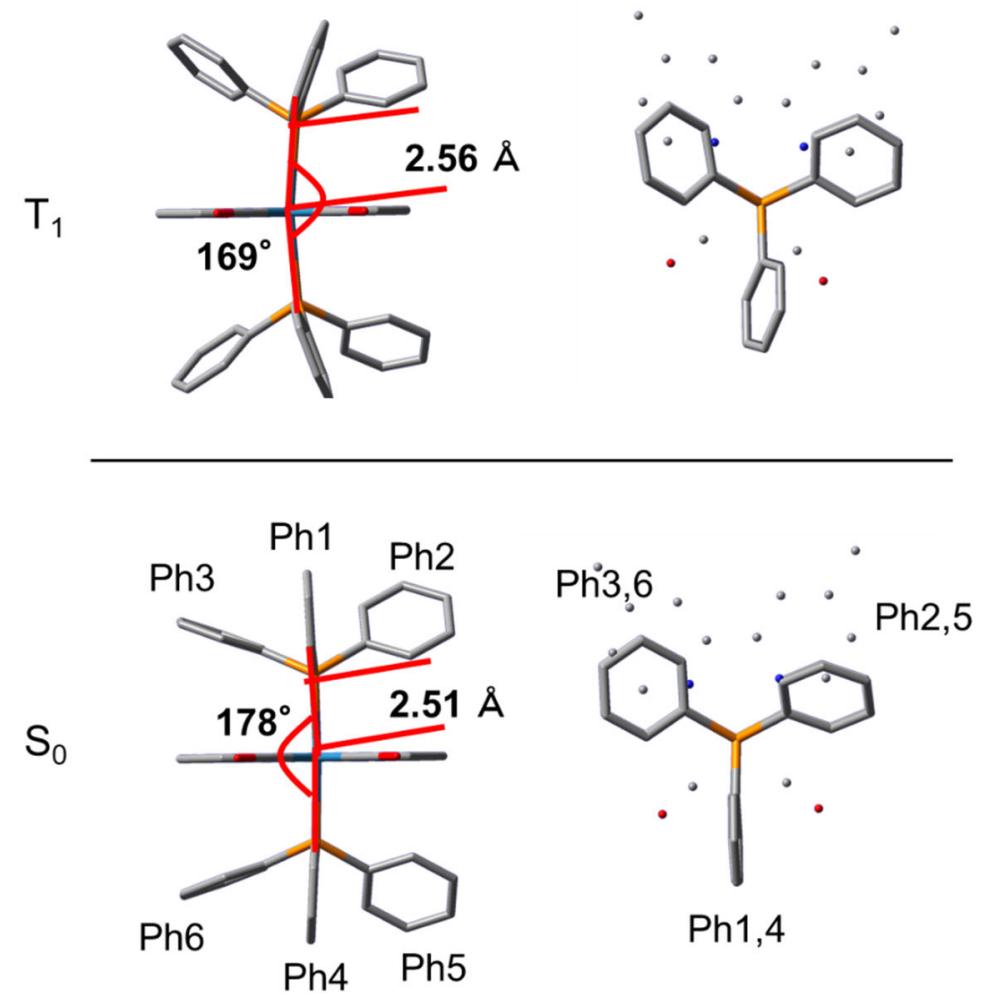
Figure 15.

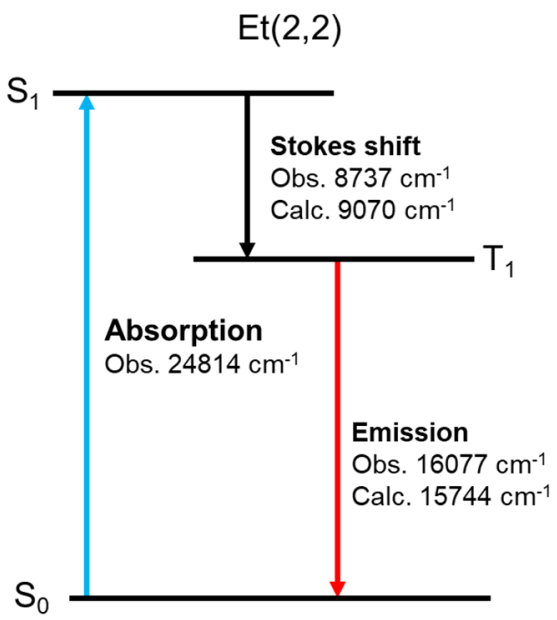

$(3,3)$

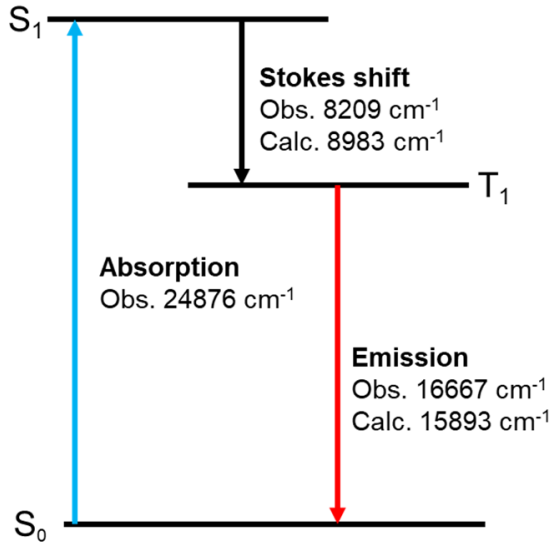


Figure 16.

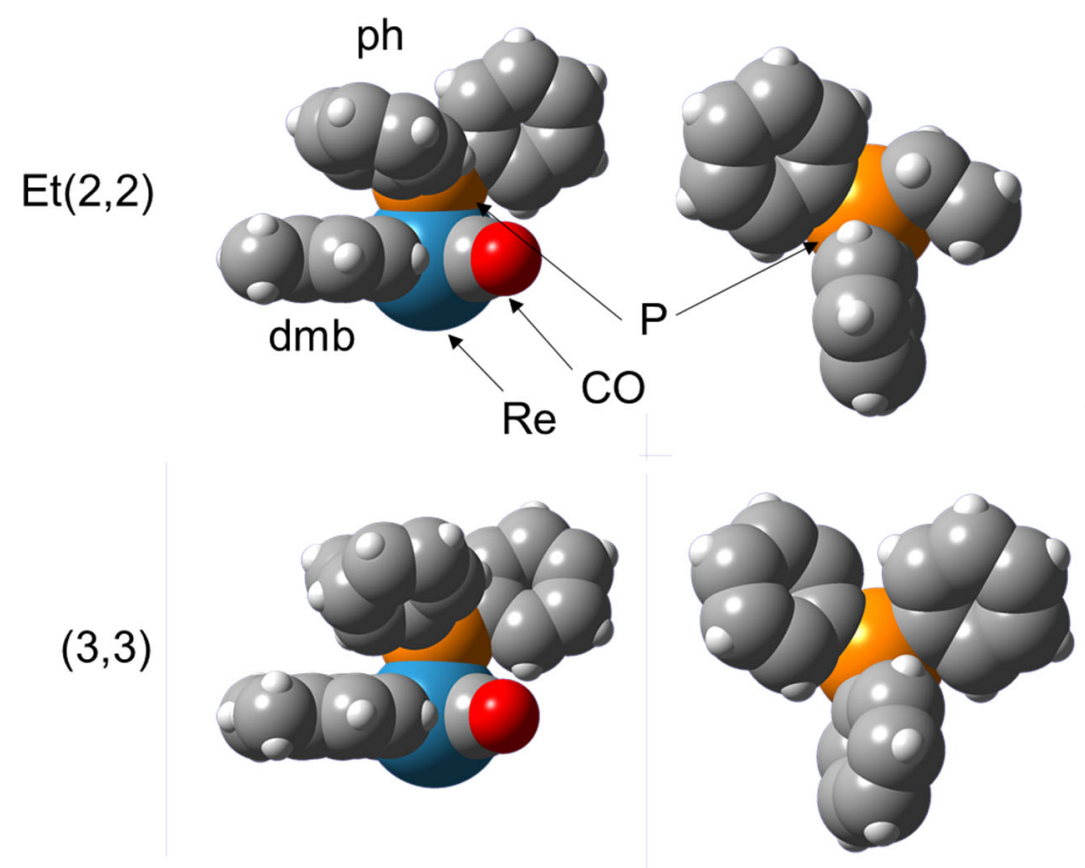


Figure 17.

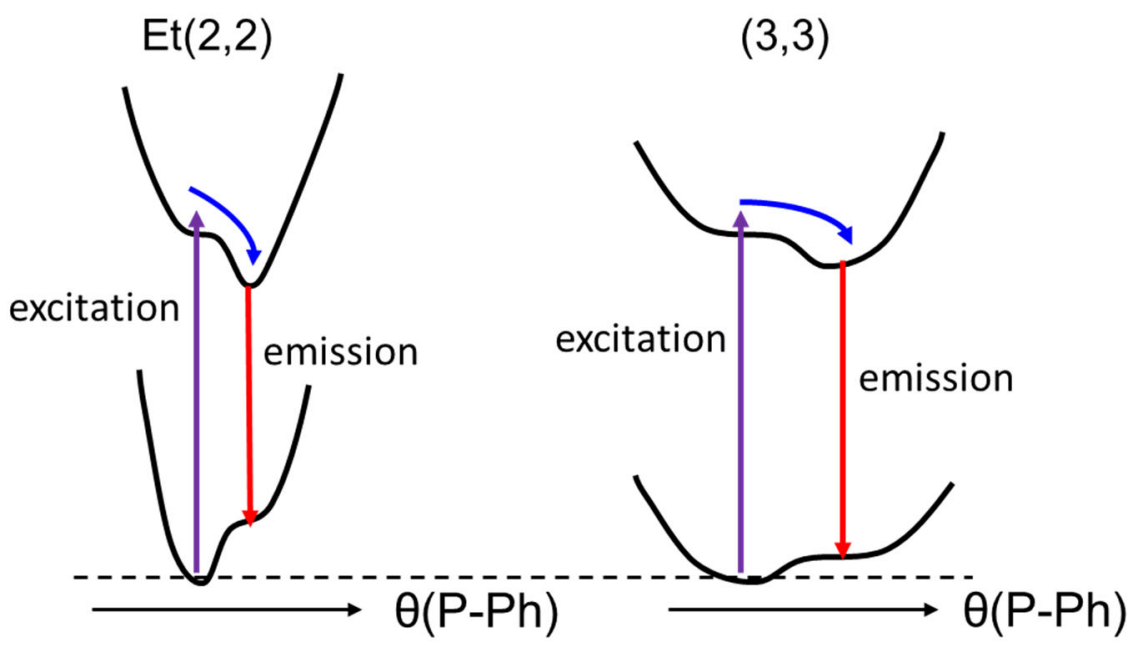


Figure 18.

(a)

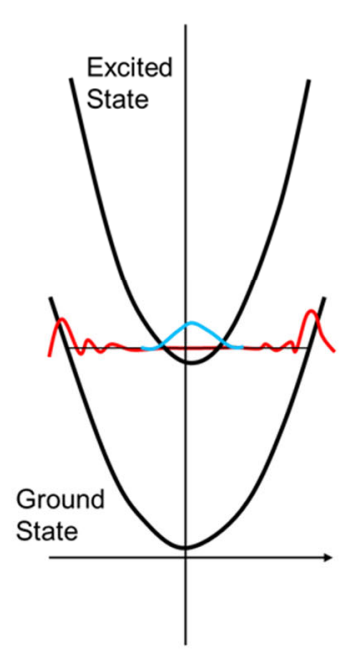

(b)

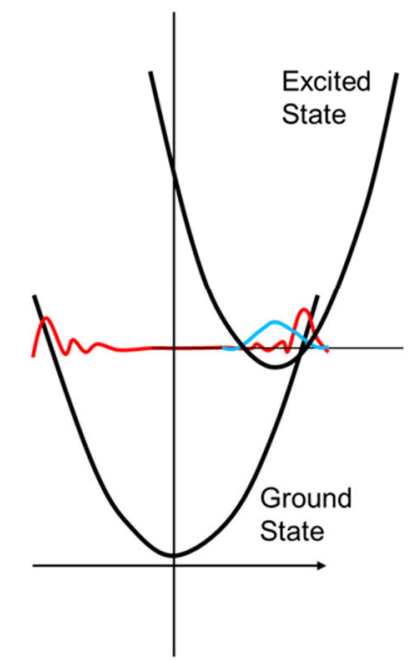

(c)

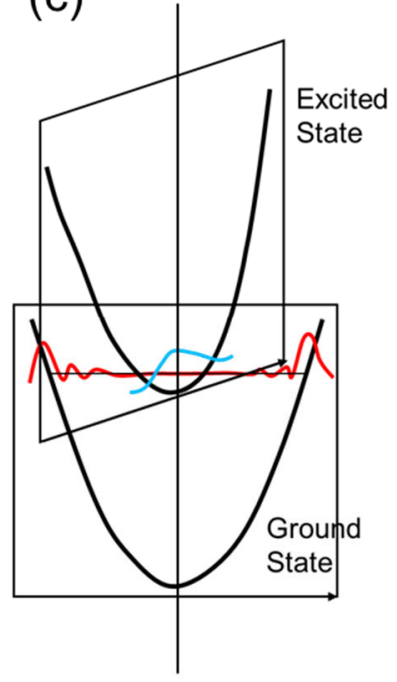




\section{Supporting Information}

\section{Determining Excited-State Structures and Photophysical Properties in Phenylphosphine Rhenium(I) Diimine Biscarbonyl Complexes Using Time- Resolved Infrared and X-ray Absorption Spectroscopies}

Yuushi Shimoda $^{1}$, Kiyoshi Miyata ${ }^{1}$, Masataka Funaki ${ }^{2}$, Tatsuki Morimoto ${ }^{3}$, Shunsuke Nozawa ${ }^{4, *}$, Shin-ichi Adachi ${ }^{4}$, Osamu Ishitani ${ }^{2}$, and Ken Onda ${ }^{1, *}$

${ }^{1}$ Department of Chemistry, Faculty of Science, Kyushu University, Motooka, Nishi-ku, Fukuoka 819-0395, Japan

${ }^{2}$ Department of Chemistry, School of Science, Tokyo Institute of Technology, O-okayama, Meguro-ku, Tokyo 152-8551, Japan

${ }^{3}$ Department of Applied Chemistry, School of Engineering, Tokyo University of Technology, Katakuramachi, Hachioji City, Tokyo 192-0982, Japan.

${ }^{4}$ Photon Factory, Institute of Materials Structure Sciences, High Energy Accelerator Research Organization (KEK), Oho, Tsukuba, Ibaraki 305-0801 Japan

*Corresponding authors: konda@chem.kyushu-univ.jp,noz@post.kek.jp 
Figure $\mathrm{S} 1$. Typical normal vibrational modes of $\mathrm{S}_{0}$ in $c i s-\mathrm{E}(2,2)$.

(a)

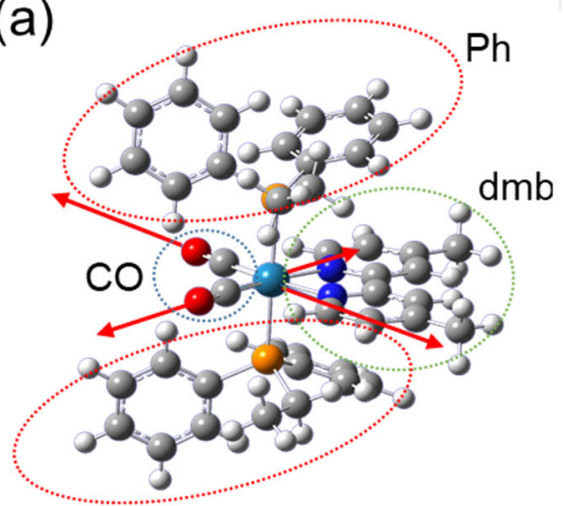

CO vibration mode

Calc. $1936 \mathrm{~cm}^{-1}$

Obs. $1923 \mathrm{~cm}^{-1}$

(c)

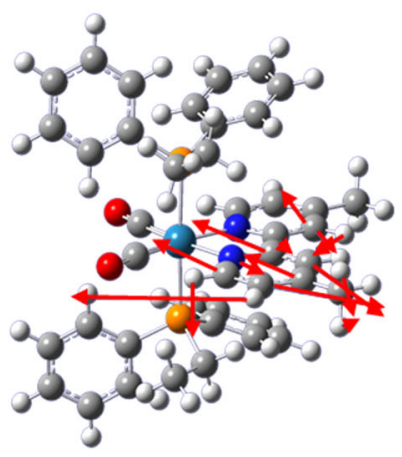

$\mathrm{dmb}$ vibration mode

Calc. $1623 \mathrm{~cm}^{-1}$

Obs. $1616 \mathrm{~cm}^{-1}$ (b)

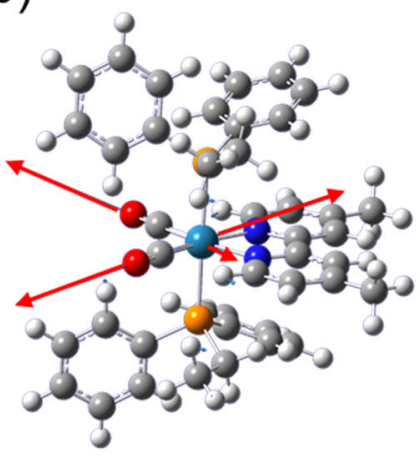

CO vibration mode

Calc. $1876 \mathrm{~cm}^{-1}$

Obs. $1850 \mathrm{~cm}^{-1}$

(d)

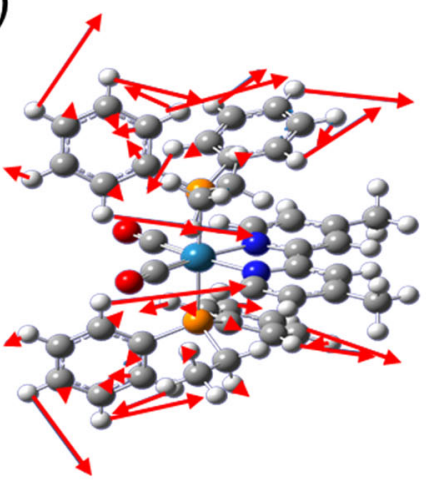

Ph vibration mode Calc. $1077 \mathrm{~cm}^{-1}$ Obs. $1096 \mathrm{~cm}^{-1}$ 
Figure S2. Typical normal vibrational modes of $\mathrm{T}_{1}$ in cis- $\mathrm{E}(2,2)$.

(a)

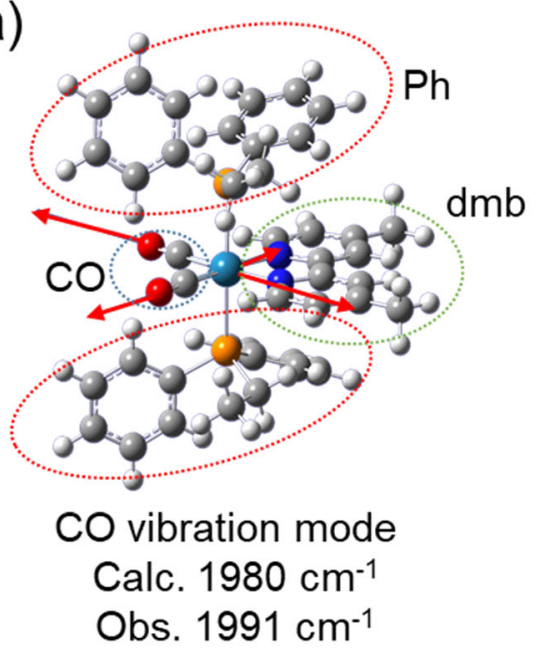

(c)

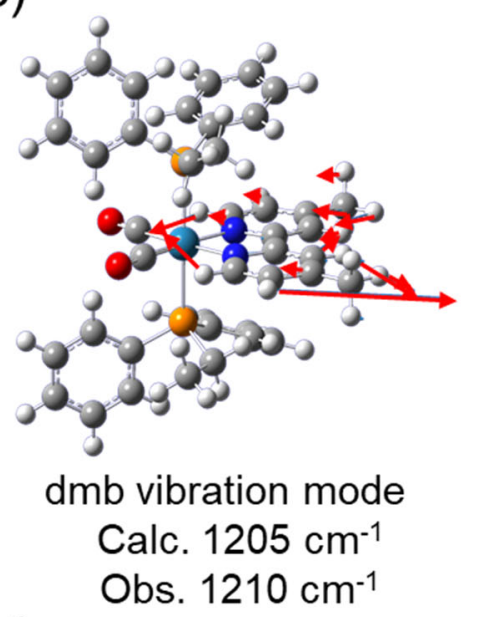

(d)

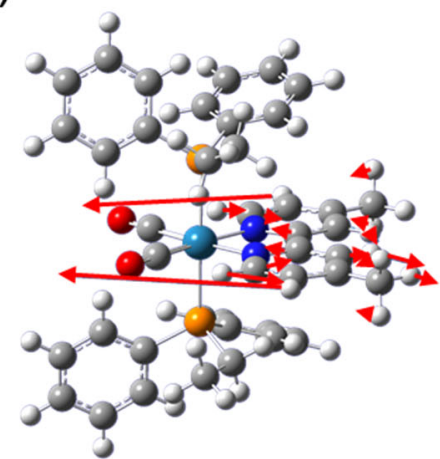

dmb vibration mode

Calc. $1100 \mathrm{~cm}^{-1}$

Obs. $1112 \mathrm{~cm}^{-1}$ (b)

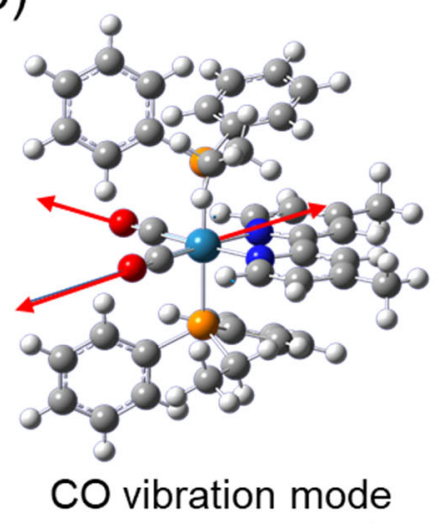

Calc. $1892 \mathrm{~cm}^{-1}$

Obs. $1899 \mathrm{~cm}^{-1}$

(e)

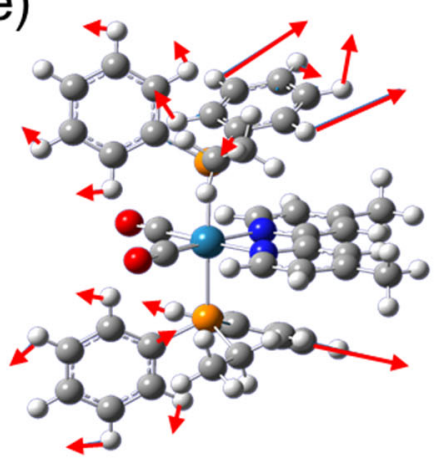

$\mathrm{Ph}$ vibration mode

Calc. $1088 \mathrm{~cm}^{-1}$

Obs. $1098 \mathrm{~cm}^{-1}$ 
Figure S3. Typical normal vibrational modes of $\mathrm{S}_{0}$ in $(3,3)$.

(a)

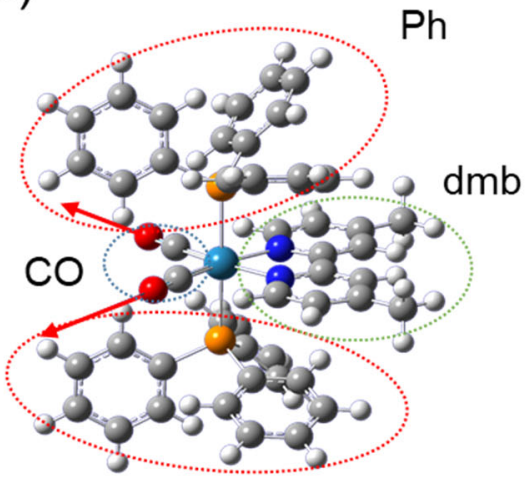

CO vibration mode

Calc. $1932 \mathrm{~cm}^{-1}$

Obs. $1933 \mathrm{~cm}^{-1}$

(c)

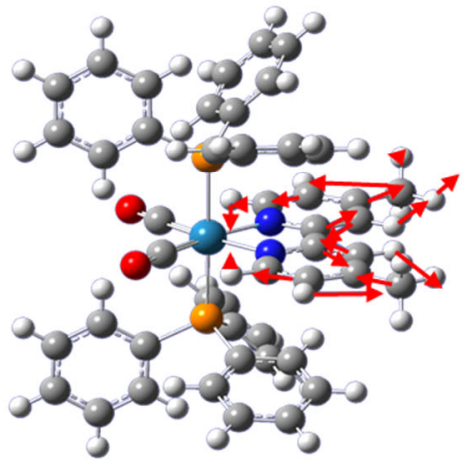

$\mathrm{dmb}$ vibration mode

Calc. $1625 \mathrm{~cm}^{-1}$

Obs. $1618 \mathrm{~cm}^{-1}$ (b)

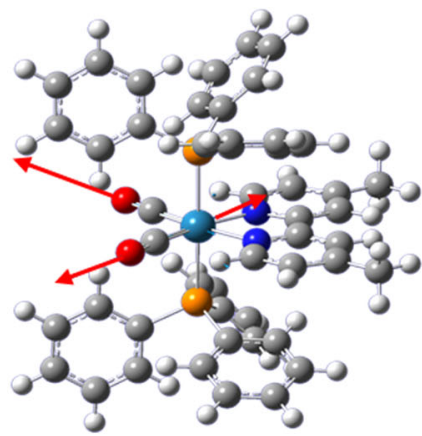

CO vibration mode

Calc. $1872 \mathrm{~cm}^{-1}$

Obs. $1850 \mathrm{~cm}^{-1}$

(d)

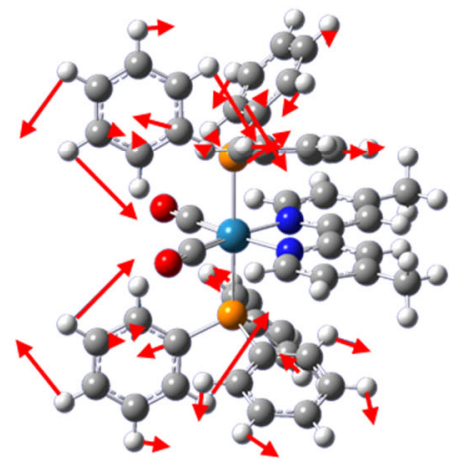

$\mathrm{Ph}$ vibration mode

Calc. $1083 \mathrm{~cm}^{-1}$

Obs. $1092 \mathrm{~cm}^{-1}$ 
Figure $\mathrm{S} 4$. Typical normal vibrational modes of $\mathrm{T}_{1}$ in $(3,3)$.

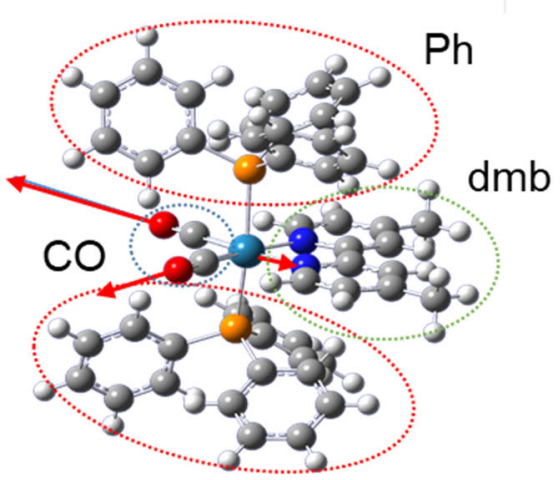

CO vibration mode

Calc. $1974 \mathrm{~cm}^{-1}$

Obs. $1986 \mathrm{~cm}^{-1}$

(c)

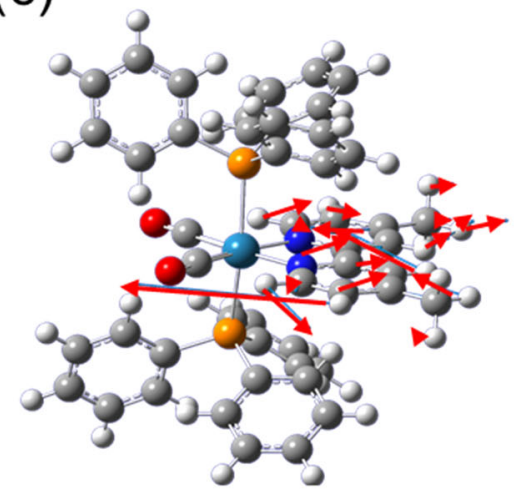

$\mathrm{dmb}$ vibration mode

Calc. $1206 \mathrm{~cm}^{-1}$

(d) Obs. $1217 \mathrm{~cm}^{-1}$

(d)

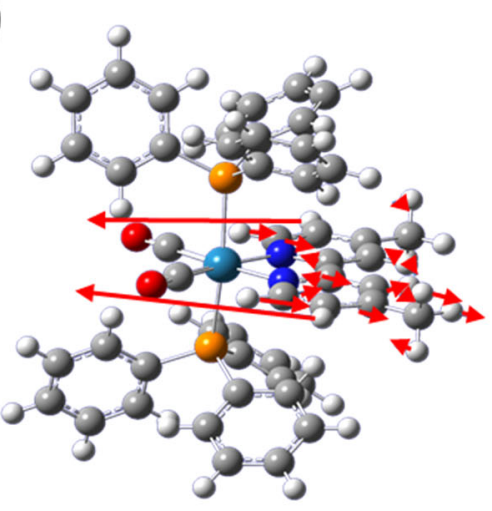

dmb vibration mode

Calc. $1102 \mathrm{~cm}^{-1}$

Obs. $1111 \mathrm{~cm}^{-1}$

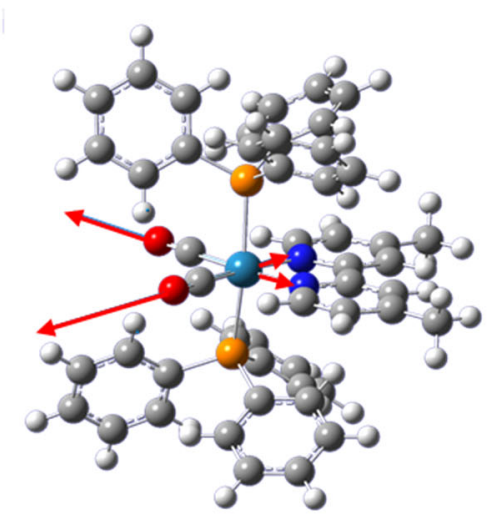

CO vibration mode

Calc. $1894 \mathrm{~cm}^{-1}$

Obs. $1905 \mathrm{~cm}^{-1}$

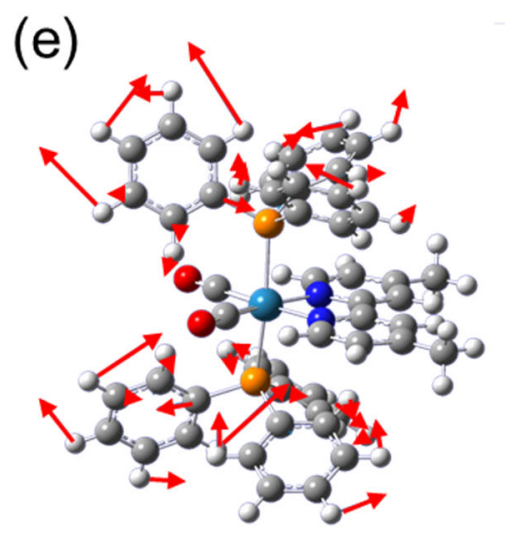

Ph vibration mode

Calc. $1084 \mathrm{~cm}^{-1}$

Obs. $1093 \mathrm{~cm}^{-1}$ 
Figure S5. Comparison of TR-IR spectra of $(3,3)$ and $(3 \mathrm{D}, 3 \mathrm{D})$.

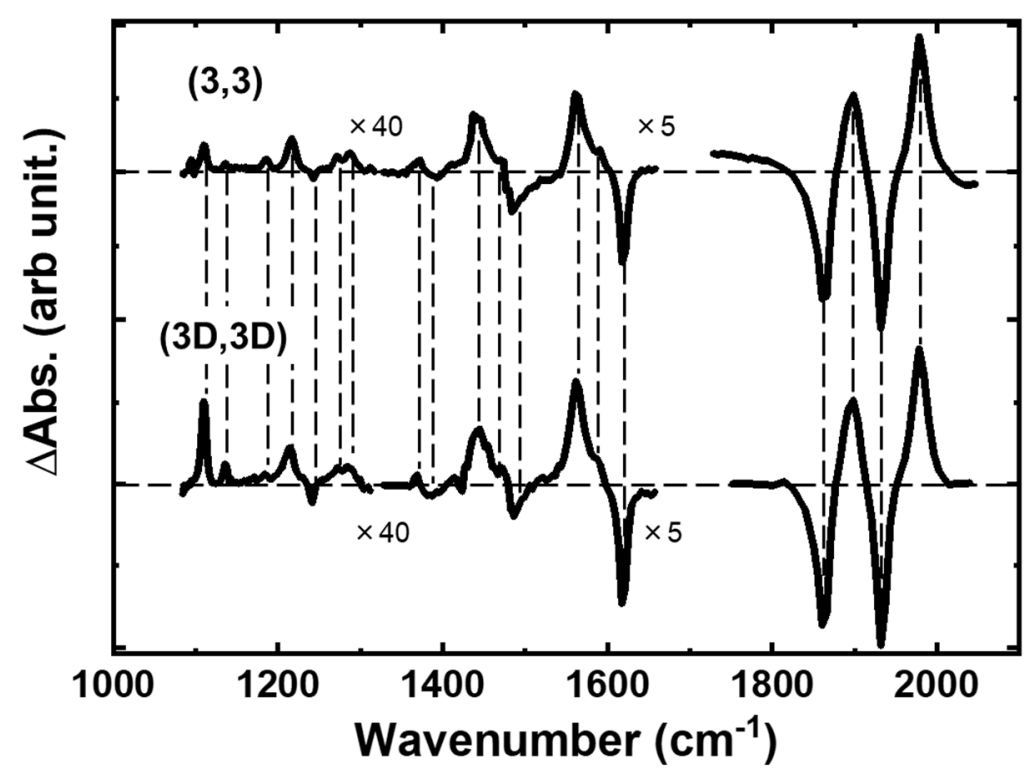


Figure S6. Comparison of observed FT-IR and TR-IR spectra of (a) Et(2,2) with the calculated spectra of the (b) cis- and (c) trans-Et $(2,2)$ geometries.

\section{Et(2,2) FT-IR}

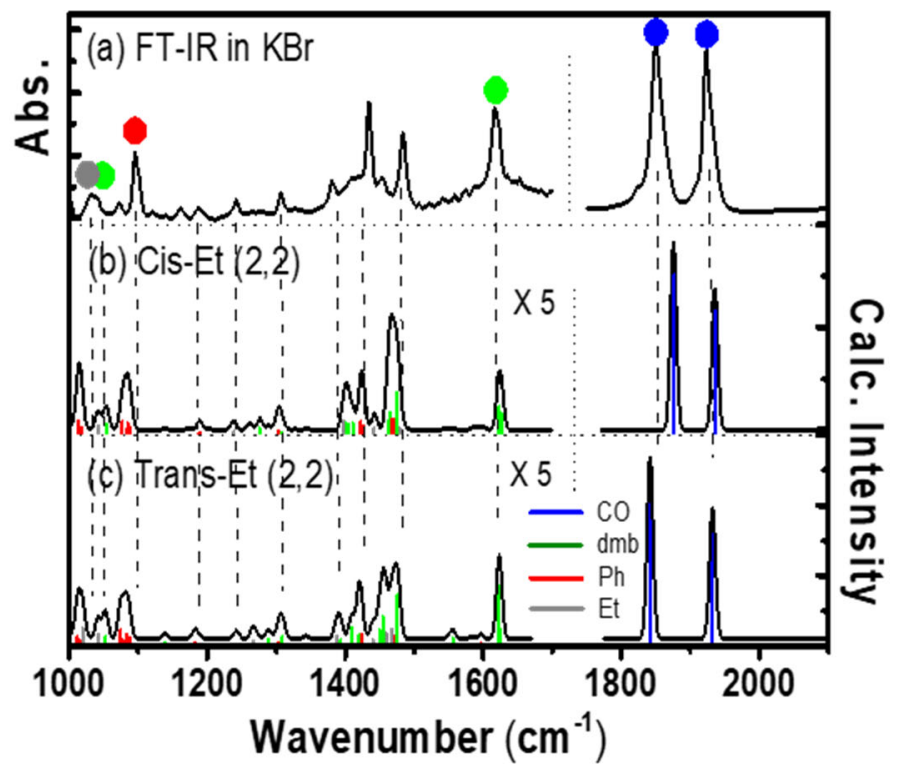

\section{Et(2,2) TR-IR}

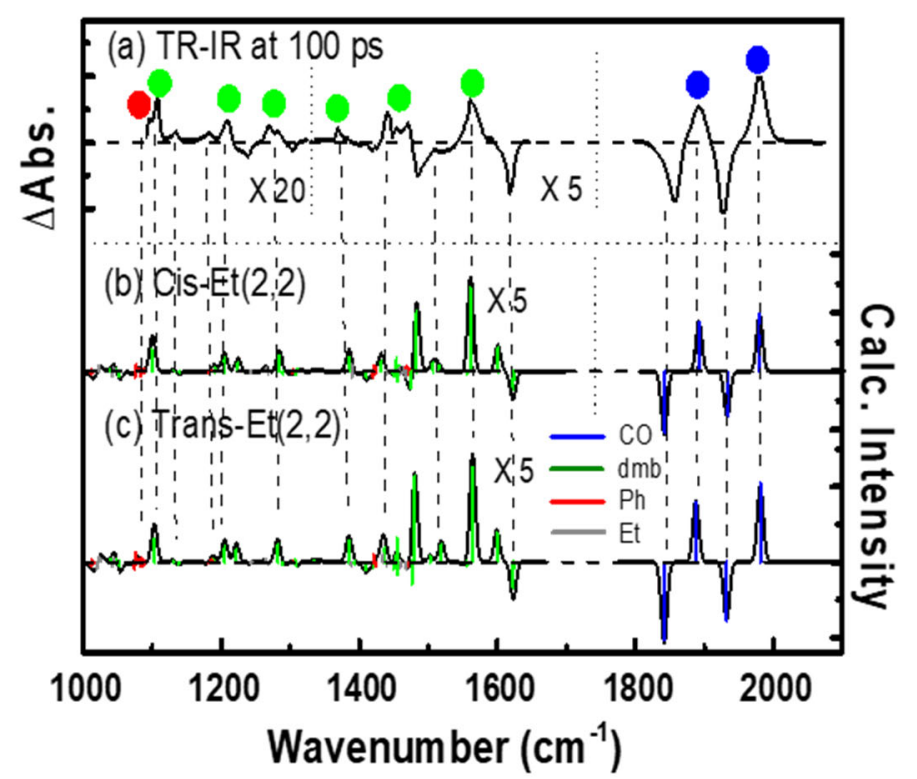


Table S1. Selected bond lengths and dihedral angles in the optimized geometries of $S_{0}$ and $T_{1}$ in cis-Et $(2,2)$. Atom labels are shown in the schematic below.

\begin{tabular}{cccc}
\hline Bond lengths $/ \AA$ & $\mathrm{S}_{0}$ calc. & $\mathrm{T}_{1}$ calc. & Difference \\
\hline Re-C1 & 1.89 & 1.96 & 0.07 \\
$\operatorname{Re}-\mathrm{C} 2$ & 1.89 & 1.90 & 0.01 \\
$\operatorname{Re}-\mathrm{P} 1$ & 2.50 & 2.54 & 0.05 \\
$\operatorname{Re}-\mathrm{P} 2$ & 2.50 & 2.54 & 0.05 \\
$\operatorname{Re}-\mathrm{N} 1$ & 2.16 & 2.14 & -0.02 \\
$\operatorname{Re}-\mathrm{N} 2$ & 2.16 & 2.05 & -0.10 \\
\hline
\end{tabular}

\begin{tabular}{cccc}
\hline Angles $/ \circ$ & $\mathrm{S}_{0}$ calc. & $\mathrm{T}_{1}$ calc. & Difference \\
\hline P1-Re-P2 & 177.6 & 169.5 & -4.6 \\
C1-Re-C2 & 93.0 & 91.9 & -2.0 \\
N1-Re-N2 & 75.1 & 77.7 & 2.8 \\
\hline
\end{tabular}

\begin{tabular}{cccc}
\hline Dihedral Angles $/{ }^{\circ}$ & $\mathrm{S}_{0}$ calc. & $\mathrm{T}_{1}$ calc. & Difference \\
\hline Ph1(P2,P1,C3,C4) & 170.2 & 168.2 & $\mathbf{- 2 . 0}$ \\
Ph2(P2,P1,C5,C6) & 95.3 & 93.7 & $\mathbf{- 1 . 6}$ \\
Ph3(P1,P2,C7,C8) & -170.2 & -168.2 & $\mathbf{2 . 0}$ \\
Ph4(P1,P2,C9,C10) & 95.3 & -93.7 & $\mathbf{1 . 6}$ \\
\hline
\end{tabular}

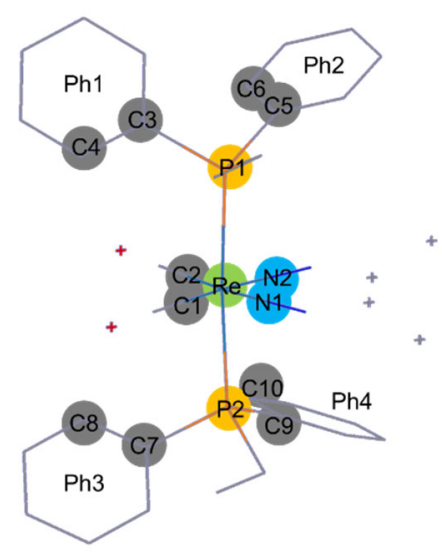


Table S2. Selected bond lengths and dihedral angles in the optimized geometries of $S_{0}$ and $T_{1}$ in $(3,3)$. Atom labels are shown in the schematic below.

\begin{tabular}{cccc}
\hline Bond lengths/ $\AA$ & $\mathrm{S}_{0}$ calc. & $\mathrm{T}_{1}$ calc. & Difference \\
\hline Re-C1 & 1.89 & 1.96 & 0.07 \\
Re-C2 & 1.89 & 1.90 & 0.01 \\
Re-P1 & 2.51 & 2.56 & 0.05 \\
Re-P2 & 2.51 & 2.56 & 0.05 \\
Re-N1 & 2.16 & 2.14 & -0.02 \\
Re-N2 & 2.15 & 2.05 & -0.01 \\
\hline & & & \\
\hline Angles / ${ }^{\circ}$ & $\mathrm{S}_{0}$ calc. & $\mathrm{T}_{1}$ calc. & Difference \\
\hline P1-Re-P2 & 177.6 & 169.5 & -8.1 \\
C1-Re-C2 & 93.0 & 91.9 & -1.0 \\
N1-Re-N2 & 75.1 & 77.7 & 2.6 \\
\hline
\end{tabular}

\begin{tabular}{cccc}
\hline Dihedral Angles $/ 0$ & $\mathrm{~S}_{0}$ calc. & $\mathrm{T}_{1}$ calc. & Difference \\
\hline Ph1(P2,P1,C3,C4) & 182.6 & 156.0 & $\mathbf{- 2 6 . 6}$ \\
Ph2(P2,P1,C5,C6) & 51.6 & 67.9 & $\mathbf{1 6 . 3}$ \\
Ph3(P2,P1,C7,C8) & 96.0 & 117.1 & $\mathbf{2 1 . 1}$ \\
Ph4(P1,P2,C9,C10) & 177.0 & 204.0 & $\mathbf{2 7 . 0}$ \\
Ph5(P1,P2,C11,C12) & -51.9 & -67.8 & $\mathbf{- 1 6 . 0}$ \\
Ph6(P1,P2,C13,C14) & -95.8 & -117.1 & $\mathbf{- 2 1 . 3}$ \\
\hline
\end{tabular}

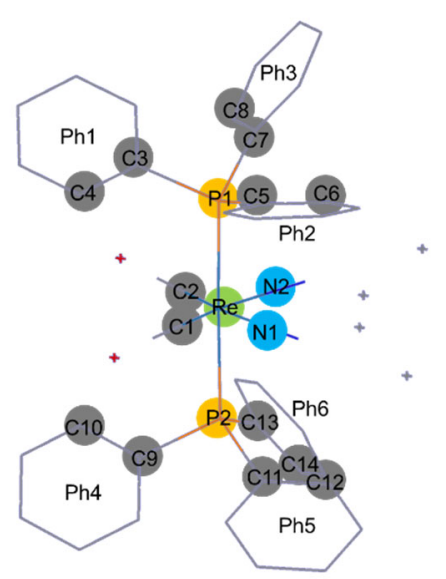




\section{Details of EXAFS Analysis}

The wavenumber $(k)$ ranges in the Fourier transformation were 3 to $12 \AA^{-1}$, as shown in the windows in Figure 14a and 14c. The Fourier transformed EXAFS functions $k^{3} \times f(k)$ of the $\mathrm{S}_{0}$ and $T_{1}$ states at $100 \mathrm{ps}$ are shown as lines in Figure $14 \mathrm{~b}$ and $14 \mathrm{~d}$. The curve-fitting analysis was performed in R space using the single scatterings from the first nearest neighbor (NN) (Re-C in $\mathrm{CO}, \mathrm{Re}-\mathrm{N}$ in dmb, and Re-P in $\mathrm{PPh}_{3}$ ), the single scatterings from the second $\mathrm{NN}$ ( $\mathrm{Re}-\mathrm{C}$ in dmb and $\mathrm{Re}-\mathrm{O}$ in $\mathrm{CO}$ ), and the multiple scatterings in $\mathrm{dmb}$ and $\mathrm{CO}$. The $\mathrm{R}$ range employed in the curve-fitting analysis was $\Delta \mathrm{R} \sim 1-3.2 \AA$, as shown in the windows in Figure $14 \mathrm{~b}$ and $14 \mathrm{~d}$. The scattering amplitudes and phase shifts were calculated by the FEFF 6 code [1] from the DFT orientations. Throughout the curve-fitting analysis, the parameters of the coordination numbers were held constant at the same values as the composition formula. For both $\mathrm{S}_{0}$ and $\mathrm{T}_{1}$, an energy origin for EXAFS $\left(E_{0}\right)$ was determined to be $10533 \mathrm{eV}$ from the inflection point obtained by the first derivative of the Re-LIII absorption edge of the $\mathrm{S}_{0}$ spectrum. For all scattering paths in $\mathrm{S}_{0}$ and $\mathrm{T}_{1}$, the same amplitude reduction factor $\left(S_{0}^{2}\right)$ and energy shift $\left(\Delta E_{0}\right)$ from $E_{0}$ were applied. The bond length of each scattering path was expressed by an expansion factor $(\alpha)$ and half path distance (reff) from the DFT orientation as $\alpha \times r e f f$. For the scattering paths from the dmb, $\mathrm{CO}$, and $\mathrm{PPh}_{3}$ ligands, different $\alpha$ and different mean-square displacements (DMS) $\left(\sigma^{2}\right)$ were considered as $\alpha_{d m b}, \alpha_{C O}, \alpha_{P}$, and $\sigma_{d m b}^{2}, \sigma_{C O}^{2}, \sigma_{P}^{2}$. For the DMS in multi-scattering paths, $\sigma_{m u l t i}^{2}$ was used as the fitting parameter. Data analysis was performed by the Larch software package [2] using a Python library.

In regards to $\mathrm{S}_{0}$, a normal EXAFS analysis was performed using the above parameters as shown in Figure 14a and 14b. The results are shown in Table S3.

The $\mathrm{T}_{1}$ EXAFS spectrum $f(k, \tau)_{T_{1}}$ was obtained by the expression

$$
f(k, \tau)_{T_{1}}=F(\tau) \Delta f(k, \tau)+f(k)_{S_{0}}
$$

where $F(\tau)$ is the scale factor at the delay time $\tau, f(k)_{T_{1}}$ is the absorption spectrum of the $\mathrm{S}_{0}$ ground state, and $\Delta f(k, \tau)$ is the difference absorption spectrum at $\tau$. To obtain $F(\tau)$ at $\tau=$ $100 \mathrm{ps}$, the minimum R-factor, which indicates the closeness-of-fit [3], was explored to give the values of $F(\tau)$ and $\Delta E_{0}$ [4,5]. From the mapping of R-factors for $F(\tau)$ and $\Delta E_{0}$ shown in Figure S7, the minimum R-factor was obtained at $F(\tau)=0.014$ and $\Delta E_{0}=13 \mathrm{eV}$. In this case, the fraction of $\mathrm{T}_{1}$ at $\tau=100 \mathrm{ps}$ was estimated to be $1.4 \%$. The EXAFS spectrum of the $\mathrm{T}_{1}$ state shown in Figure $14 \mathrm{c}$ was derived by eq. (1) with $F(\tau)=0.014$. The EXAFS analysis for $\mathrm{T}_{1}$ was performed using the same parameters in $\mathrm{S}_{0}$. Table $\mathrm{S} 3$ shows the fitting results for $\mathrm{T}_{1}$. Although the valence of $\mathrm{S}_{0}$ is $\operatorname{Re}(\mathrm{I})$, the valence of $\mathrm{T}_{1}$ is $\operatorname{Re}(\mathrm{II})$ because it is a transient charge transfer state. Therefore, in the EXAFS fitting result, the $\Delta E_{0}$ of $\mathrm{T}_{1}$ is larger than that of $\mathrm{S}_{0}$ because of the effect of chemical shift. 
Figure S7. R-factor mapping for $\mathrm{F}(\tau)$ and $\Delta \mathrm{E}_{0}$ in the EXAFS analysis of $\mathrm{T}_{1}$ in $(3,3)$.

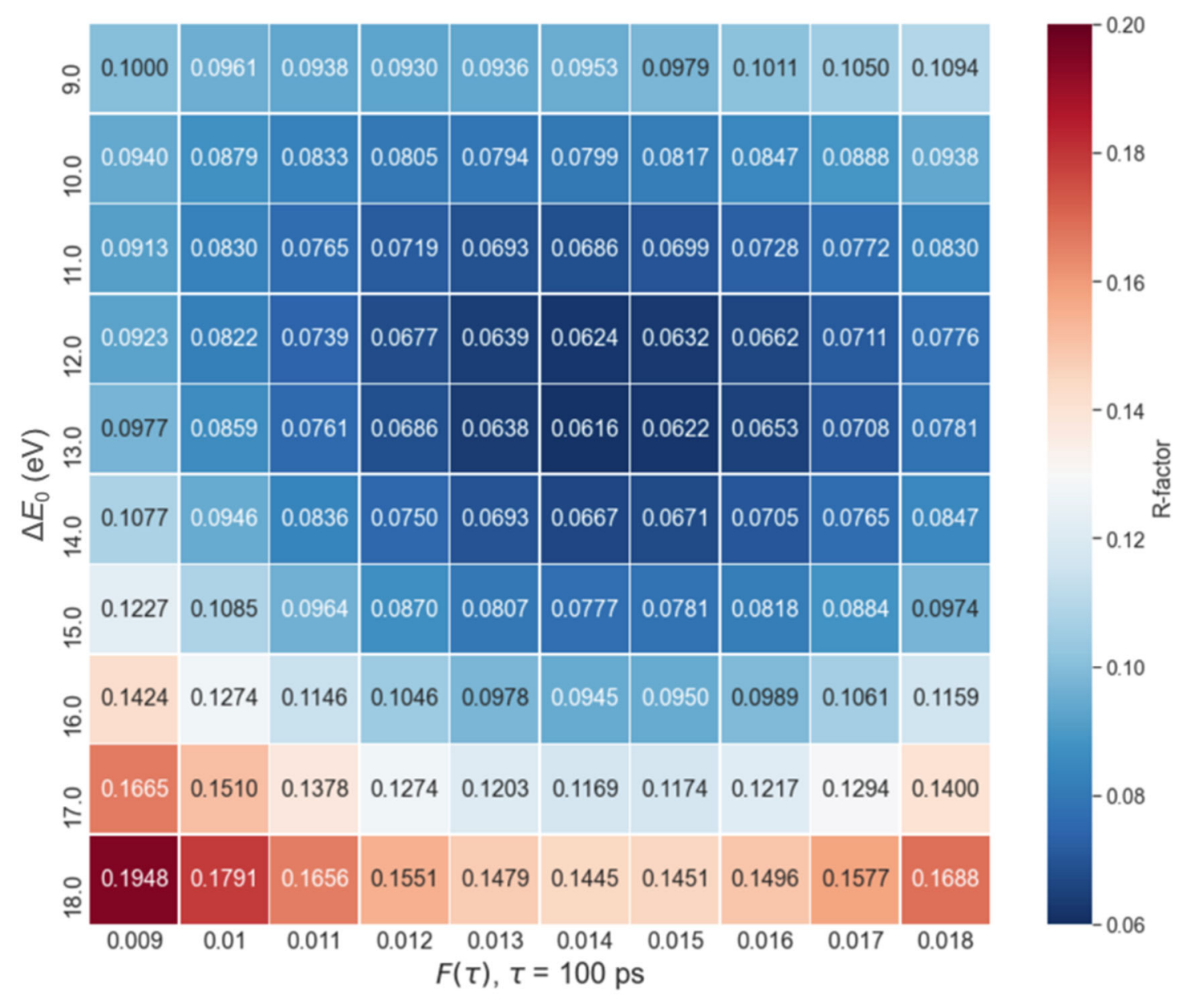


Table S3. Fitting results from the EXAFS analysis of $\mathrm{S}_{0}$ and $\mathrm{T}_{1}$ in $(3,3)$.

\begin{tabular}{ccc}
\hline & $\mathrm{S}_{0}$ & $\mathrm{~T}_{1}$ \\
\hline R-factor & 0.029 & 0.061 \\
$S_{0}{ }^{2}$ & $1.0(3)$ & $1.4(5)$ \\
$\Delta E_{0}$ & $5.0(27)$ & $12.6(21)$ \\
$\alpha_{d m b}$ & $-0.012(13)$ & $-0.013(7)$ \\
$\alpha_{C O}$ & $-0.013(6)$ & $-0.003(6)$ \\
$\alpha_{P}$ & $-0.039(7)$ & $-0.005(6)$ \\
$\sigma_{d m b}{ }^{2}$ & $0.004(4)$ & $0.001(1)$ \\
$\sigma_{C O}{ }^{2}$ & $0.006(3)$ & $0.015(13)$ \\
$\sigma_{P}{ }^{2}$ & $0.004(2)$ & $0.005(3)$ \\
$\sigma_{\text {multi }}{ }^{2}$ & $0.004(2)$ & $0.003(3)$ \\
\hline
\end{tabular}




\section{References}

[1] Zabinsky, S. I.; Rehr, J. J.; Ankudinov, A.; Albers, R. C.; Eller, M. J., Multiple-scattering calculations of x-ray-absorption spectra. Phys Rev B Condens Matter 1995, 52 (4), 2995-3009.

[2] Newville, M. In Larch: an analysis package for XAFS and related spectroscopies, 2013; p 012007.

[3] Calvin, S., XAFS for Everyone. CRC press: 2013.

[4] Gawelda, W.; Pham, V. T., RM van der Veen, D. Grolimund, R. Abela, M. Chergui, C. Bressler. J. Chem. Phys 2009, 130, 124520.

[5] Sato, T.; Nozawa, S.; Tomita, A.; Hoshino, M.; Koshihara, S.; Fujii, H.; Adachi, S., Coordination and electronic structure of ruthenium (II)-tris-2, $2^{\prime}$-bipyridine in the triplet metalto-ligand charge-transfer excited state observed by picosecond time-resolved Ru K-edge XAFS. The Journal of Physical Chemistry C 2012, 116 (27), 14232-14236. 